Pacific Journal of Mathematics

SOLUBILITY OF FINITE GROUPS ADMITTING A
FIXED-POINT-FREE AUTOMORPHISM OF ORDER $r$ st. I 


\title{
SOLUBILITY OF FINITE GROUPS ADMITTING A FIXED-POINT-FREE AUTOMORPHISM OF ORDER rst I
}

\author{
Peter ROWLey
}

\begin{abstract}
The 'fixed-point-free automorphism conjecture' asserts that if a finite group $G$ admits a fixed-point-free automorphism group $A$ (and, if $A$ is noncyclic, further suppose that $(|G|,|A|)=1$ ), then $G$ is soluble. This paper is the first in a four part series, which considers the above conjecture when $A$ is cyclic of order $r s t$ where $r, s$ and $t$ are distinct prime numbers.
\end{abstract}

1. Introduction. Suppose $G$ is a finite group. For $A$ a subgroup of the automorphism group of $G$ we say that $A$ acts fixedpoint-freely upon $G$ if and only if $C_{G}(A)=\{g \in G \mid a(g)=g, \forall a \in A\}=\{1\}$. When $A=\langle\alpha\rangle$ is cyclic we sometimes say $\alpha$ acts fixed-point-freely upon $G$.

Let $r, s$ and $t$ denote distinct prime numbers. The main result to be proved here is

THEOREM 1.1. A finite group which admits a coprime fixedpoint-free automorphism of order rst is soluble.

In [15] the above result is obtained with the additional assumption that $r s t$ is a non-Fermat number (for the definition of a nonFermat number see $\S 4$ ). The main result of [15] has been further extended in [17] where the 'fixed-point-free automorphism conjecture' is established for automorphisms whose order is a non-Fermat squarefree number. The 'fixed-point-free automorphism conjecture' asserts the following.

If a finite group $G$ admits a fixed-point-free automorphism group $A$ (and, if $A$ is noncyclic, further suppose that $(|G|,|A|)=1$ ), then $G$ is soluble.

References for other works which contribute to the solution of this problem may be found in [13] and [16].

We now review the strategy of the proof of Theorem 1.1. A substantial part of our arguments will be in the context of a minimal situation. So let the pair $(G,\langle\alpha\rangle)$ be a counterexample to Theorem 1.1 chosen so that $|G|+|\langle\alpha\rangle|$ is minimal. Lemma 3.13 demonstrates, in such a group, the existence of certain $\alpha$-invariant nilpotent Hall subgroups. Let $L$ and $M$ denote (respectively) $\alpha$-invariant nilpotent Hall $\lambda$ - and $\mu$-subgroups of $G$. By (2.22) the number of maximal 
$\alpha$-invariant $\{\lambda \cup \mu\}$-subgroups of $G$ is at most two. Making use of this fact, for various choices of $L$ and $M$, we deduce the possible consequences when $L$ and $M$ do not permute. Such deliberations are sometimes referred to as 'local analysis'. The structural consequences predicted by the local analysis must, in some way, be woven together to present 'global' information about $G$. Frequently the transition from local to global information in this type of problem is achieved by factorizing $G$ as the product of two proper $\alpha$-invariant subgroups. In [13] and [14] the local information obtained leads fairly naturally to a 'useful' factorization. In proving Theorem 1.1 there arise many more possible interactions globally (that is, the local deductions are not as restrictive as in [13] and [14]). The diverse possibilities (globally) force us to consider the interaction between more than just a pair of $\alpha$-invariant nilpotent Hall subgroups. We have termed results dealing with such situations as 'linking theorems'. Valuable contributions to the proofs of the linking theorems are made by (2.6), (2.14), (2.26), the (so-called) triangle lemmas and Theorem 4.4. Let $P$ denote the (unique) $\alpha$-invariant Sylow $p$-subgroup of $G$. We say $P$ is of type $\Delta$ where $\Delta \subseteq \Lambda=\{1,2,3\}$ according to the triviality or otherwise of $C_{P}\left(\alpha^{j}\right)$ for various $j$ (a precise definition is enunciated in $\S 3$ ). Depending on whether $\Delta=\Lambda$ or $\Delta \neq \Lambda$ the 'nuts and bolts' of the proofs of certain technical lemmas will differ. For example, if we have two $\alpha$-invariant Sylow subgroups of types (say) $\Delta$ and $\Gamma$ with $\Delta \neq \Lambda \neq \Gamma$, then results such as (2.11) are available. Whilst, if $\Delta=\Lambda=\Gamma$, then the two $\alpha$-invariant Sylow subgroups will have more interaction between their $\alpha$-invariant subgroups (see for instance Lemma 3.14), which, sometimes, may be exploited to advantage. A further general point is that, unlike most current work on nonsoluble finite groups, during the proof of Theorem 1.1 we have few encounters with centralizers of involutions. However, a fortuitous meeting in the proofs of Theorem 8.6 and Lemma 14.10 helps us out of a potential empasse.

The proof of Theorem 1.1 will be presented in four parts; our section numbering will run through all the parts. The material presented in Part $I$ is of a more general nature and also covers much of the groundwork for [17]. Section 2 establishes notation and reviews results from other sources. In $\S 3$ we introduce the concept of the 'star-subgroup' and the 'type' of an $\alpha$-invariant Sylow subgroup. The star subgroup figures prominently in $\S 4$ where we study soluble groups which admit a fixed-point-free automorphism. Section 5 contains certain preliminary observations pertinent to the linking theorems, and includes the 'triangle lemmas'. Some criteria for normal $p$-complements are noted in $\S 6$. In $\S 7$, contained in Part II, we begin the proof of Theorem 1.1 in earnest; $\S 7$ catalogues the 
local information relevant to Theorem 1.1. The remaining two sections of Part II are devoted to establishing various linking theorems.

The sequel to Part II is wholly concerned with showing that the $\alpha$-invariant Sylow subgroups of type $\Lambda=\{1,2,3\}$ (in a minimum counterexample) generate an $\alpha$-invariant soluble subgroup. It is left to Part IV to examine the various factorizations that arise and draw together the threads of the proof, from which we infer that no counterexample can exist.

During the course of this work I enjoyed the stimulating hospitality of the Mathematics Institute at the University of Warwick. Also, I would like to thank Dr. Trevor Hawkes for supplying the example following Theorem 4.4, and Jenny, my wife, without whose financial and moral support this work would never have seen the light of day.

2. Assumed results and notation. The notation of [7] will be adhered to as much as possible, and all groups considered in this paper will be assumed to be finite.

The first result summarizes certain well-known properties germane to fixed-point-free automorphisms which will be used frequently. When $A$ is cyclic, (2.1)(i) is proved in Lemma 10.1.3 of [7] and, when $A$ is noncyclic, (2.1)(i) follows from Theorem 6.2.2 of [7]. For verification of the remainder of $(2.1)$ see $[(2.1) ; 16]$.

(2.1) Let $G$ be a finite group admitting a fixed-point-free automorphism group $A$ (and if $A$ is noncyclic also assume $(|G|,|A|)=1$ ). Then

(i) If $N$ is a normal $A$-invariant subgroup of $G$, then $A$ acts fixed-point-freely on $G / N$.

(ii) For each $p \in \pi(G)$ there exists a unique $A$-invariant Sylow $p$-subgroup, $P$, of $G$ and, moreover, $P$ contains every $A$-invariant $p$-subgroup of $G$;

(iii) If $H$ is an $A$-invariant subgroup of $G$ and $P$ is the (unique) $A$-invariant Sylow $p$-subgroup of $G$, then $H \cap P$ is the (unique) $A$ invariant Sylow $p$-subgroup of $H$.

(iv) If $G$ possesses an $A$-invariant Hall $\pi$-subgroup $H$, then $H$ contains all $A$-invariant $\pi$-subgroups of $G$. Also, if $K$ is an $A$ invariant subgroup of $G$, then $K \cap H$ is the $A$-invariant Hall $\pi$-subgroup of $K$.

(v) If $G$ is soluble, then, for each set $\pi$ of primes, there exists an $A$-invariant Hall $\pi$-subgroup of $G$ which (by (iv)) is unique and contains every $A$-invariant $\pi$-subgroup of $G$.

(2.2) Suppose $G$ is a finite group admitting the automorphism 
$\alpha$ fixed-point-freely.

(i) (Thompson [19]). If $|\langle\alpha\rangle|$ is a prime, then $G$ is nilpotent.

(ii) (Ralston [14]). If $|\langle\alpha\rangle|=r s$ (where $r$ and $s$ are distinct primes), then $G$ is soluble.

Our next result is a compendium of results pertaining to coprime operator groups.

(2.3) Let $G$ be a finite group admitting $A$ as a coprime operator group.

( i ) $G=C_{G}(A)[G, A]$ where $[G, A]=\left\langle g^{-1} \alpha(g) \mid g \in G, \alpha \in A\right\rangle$. Moreover, if $G$ is abelian, then $G=C_{G}(A) \times[G, A]$. $G$.

(ii ) $[[G, A], A]=[G, A]$ is an $A$-invariant normal subgroup of

(iii) If $N$ is a normal $A$-invariant subgroup of $G$, then $C_{G / N}(A)=$ $\left(C_{G}(A) N\right) / N$.

(iv) If $A$ is an elementary abelian $r$-group ( $r$ a prime) of rank 2 , then $G=\left\langle C_{G}(\alpha) \mid \alpha \in A^{\sharp}\right\rangle$.

( v ) Suppose $G$ is a nilpotent group with $H$ a subgroup of $G$. If $C_{G}(H) H \leqq C_{G}(A)$, then $G=C_{G}(A)$.

(vi ) If $B \leqq A$, then $C_{G}(B)$ and $[G, B]$ are $A$-invariant.

(vii) If $H$ is an $A$-invariant subgroup of $G$ containing [G, $A$ ], then $[G, A]=[H, A]$.

(viii) If $H$ is an $A$-invariant subgroup of $G$, then $C_{N_{G}(H)}(A) \leqq$ $N_{G}([H, A])$.

(ix) If $G=H K$ where $H$ and $K$ are $A$-invariant subgroups of $G$ with $K \leqq C_{G}(A)$, then $[G, A] \leqq H$ (and so, by (vii), $[G, A]=[H, A]$ ).

( $\mathrm{x})$ If $H \leqq G$ with $H \leqq C_{G}(A)$, then $[G, A]$ centralizes $H$.

(xi) Suppose $G=H K$ where $H$ and $K$ are $A$-invariant subgroups of $G$. Assume $H \leqq G$ and $(|H|,|K|)=1$, and set $\pi=\pi(H)$. If $H \leqq C_{G}(A)$ and $C_{G}(A)$ has a normal $\pi$-complement, then $[H, K]=1$.

(xii) If $H \leqq C_{G}(A)=C$, then $N_{G}(H)=N_{C}(H) C_{G}(H)$.

(xiii) For each $p \in \pi(G)$ there exists at least one $A$-invariant Sylow $p$-subgroup and any two $A$-invariant Sylow $p$-subgroups of $G$ are conjugate by an element of $C_{G}(A)$. Also, every $A$-invariant $p$ subgroup of $G$ is contained in some $A$-invariant Sylow $p$-subgroup of $G$.

(xiv) Assume $G$ is soluble and $\pi$ is a set of primes. Then $G$ possesses at least one $A$-invariant Hall $\pi$-subgroup, any two Hall $\pi$ subgroups are conjugate by an element of $C_{G}(A)$ and every $A$-invariant $\pi$-subgroup of $G$ is contained in an $A$-invariant Hall $\pi$-subgroup of G.

(xv) Suppose that $A=B C$ where $B \leqq A$ and $C \leqq A$, that $C_{G}(A)=1$ and that $G=C_{G}(B) C_{G}(C)$. Then $G=C_{G}(B) \times C_{G}(C)$. 
Proof. For parts (i)-(x) consult (2.6) of [16]; the proof of [Theorem 5.2.3; 7] may be used to show that, in (i), the sum is direct when $G$ is abelian. Part (xi) follows by combining parts (i), (ix), and (x), and part (xii) is given as Corollary 2 to Theorem 3 in [5]. For part (xiii) see Theorem 6.2.2 of [7]; taking into account [Theorem 6.4.1; 7] the proof of Theorem 6.2.2 of [7] also yields part (xiv). Part (xv) may be verified as follows. From (vi), both $C_{G}(B)$ and $C_{G}(C)$ are $A$-invariant and a double application of (ix) (with $B$ and $C$ in place of $A)$ yields that $[G, B] \leqq C_{G}(C)$ and that $[G, C] \leqq C_{G}(B)$. Since $C_{G}(A)=1, C_{G}(B) \cap C_{G}(C)=1$ whence (using (i) for $B$ and $C$ ), $C_{G}(B)=[G, C]$ and $C_{G}(C)=[G, B]$. Hence, as $[G, B] \leqq G$ and $[G, C] \leqq G$, the sum must be direct and (xv) follows.

The following two results describe solubility criteria. A proof for $(2.4)$, when $A$ is cyclic, is given in [(2.12); 14]. However, the proof also works for a noncyclic (coprime) automorphism group.

(2.4) Suppose $G$ admits the automorphism group $A$ fixed-pointfreely (with $(|G|,|A|)=1$ if $A$ is noncyclic). Then $G$ is soluble if and only if for each pair of primes $p, q \in \pi(G)$ the corresponding $A$ invariant Sylow $p$ - and $q$-subgroups of $G$ permute.

(2.5) (Wielandt [p. 680, 9]). If $G=H K$ where $H$ and $K$ are nilpotent Hall subgroups of $G$, then $G$ is soluble.

For $P$, a $p$-group, $J(P)$ denotes the Thompson subgroup of $P$, as defined in [p. 271; 7]. In establishing certain 'linking theorems' the next theorem is of great help.

(2.6) Let $G$ be a soluble group admitting $A$ fixed-point-freely and let $P$ be the $A$-invariant Sylow $p$-subgroup of $G$. If $A$ is noncyclic also assume that $(|G|,|A|)=1$. Then $G=O_{p^{\prime}}(G) N_{G}(J(P)) C_{G}(Z(P))$.

Proof. If $G \neq O_{p^{\prime}}(G) N_{G}(J(P)) C_{G}(Z(P))$, then, from the main theorem of [6],

$$
p=2 \text { or } 3 \text {; }
$$

and there exists a (nontrivial) section $H / K$ of $G$ (where $H$ and $K$ are $A$-invariant subgroups of $G$ and $K \leqq H$ ) such that $H / K$ is a direct product of copies of SL $(2, p)$.

Clearly $H / K$ admits the action of $A$ and, by (2.1)(i), the action will be fixed-point-free. Set $H / K=\bar{H}$. Then $\bar{H}=\bar{H}_{1} \times \cdots \times \bar{H}_{n}$ where $\bar{H}_{i} \cong \mathrm{SL}(2, p)$. Observe, since the orders of $Z(\mathrm{SL}(2, p))$ and SL $(2, p) / \mathrm{SL}(2, p)^{\prime}$ are coprime, that $A$ will permute the $\bar{H}_{i}$ by the 
Krull-Remak-Schmidt theorem (see [Satz 12.3, p. 66; 9]).

Set $B=N_{A}\left(\bar{H}_{1}\right)$. By (2.1)(ii), there exists an $A$-invariant Sylow 2-subgroup of $\bar{H}$, say $\bar{T}$. If $p=2$, then $\left|\bar{T} \cap \bar{H}_{1}\right|=2$ and so (when $p=2) C_{\bar{H}_{1}}(B) \neq 1$. When $p=3$, then $\left|Z\left(\bar{H}_{1}\right)\right|=2$ and so $C_{\bar{H}_{1}}(B) \neq 1$. Hence

$$
1 \neq a_{1}(\bar{h}) \cdots a_{k}(\bar{h}) \in C_{\bar{H}_{1}}(A),
$$

where $1 \neq \bar{h} \in C_{\bar{H}_{1}}(B)$ and $a_{1}, \cdots, a_{k}$ is a left transversal to $B$ in $A$, contrary to $C_{\bar{H}}(A)=1$. Therefore $G=O_{p^{\prime}}(G) N_{G}(J(P)) C_{G}(Z(P))$.

We also require a slight extension of (2.6); (2.12) of [16] furnishes a proof for (2.7).

(2.7) Let $G$ be a soluble group admitting a fixed-point-free automorphism group $A$ (with, if $A$ is noncyclic, $(|G|,|A|)=1$ ) and assume that $G$ possesses an $A$-invariant nilpotent Hall $\eta$-subgroup, $N$ say. Then

$$
G=\left\langle N_{G}(J(P)), C_{G}(Z(P)) \mid p \in \eta, P \in \operatorname{Syl}_{p}(N)\right\rangle O_{\eta^{\prime}}(G) .
$$

The next result, (2.8), appears as Theorem 4.1 of [14] for the case $|\langle\alpha\rangle|=r s$ ( $r$ and $s$ distinct primes), but the proof is valid for any $\alpha$ of square-free order; (2.9) may be established by a proof analogous to the one given in [14] for Lemma 3.5 (and does not require $\alpha$ to be of square-free order).

(2.8) (Ralston). Let $G$ be a finite group admitting a fixed-pointfree automorphism $\alpha$ of square-free order, and let $P$ denote the $\alpha$ invariant Sylow $p$-subgroup of $G$. If $C_{P}\left(\alpha^{j}\right)=1$ for all $j, 1 \leqq j<$ $|\langle\alpha\rangle|$, then $P$ is a direct summand of $G$.

(2.9) Suppose $G$ is a soluble group admitting $\alpha$ fixed-point-freely. If $R$ is an $\alpha$-invariant $p$-subgroup of $G$ ( $p$ a prime) such that $C_{R}\left(\alpha^{j}\right)=1$ for all $j, 1 \leqq j<|\langle\alpha\rangle|$, then $R \leqq O_{p}(G)$.

(2.10) Let $G$ be a group admitting the coprime fixed-point-free automorphism $\alpha$ of order $r s$ (where $r$ and $s$ are distinct primes), and set $\rho=\alpha^{s}$ and $\sigma=\alpha^{r}$. Let $P$ denote the $\alpha$-invariant Sylow $p$ subgroup of $G, p$ a prime. Then

(i) $G$ has Fitting length at most 2;

(ii) if $P=C_{P}(\rho) C_{P}(\sigma)$, then $G$ has a normal $p$-complement; and

(iii) if $p \neq 2$, then $P=C_{P}(\rho) C_{P}(\sigma) O_{p}(G)$.

Proof. (i) From (2.2)(ii) $G$ is soluble and so, by the main result of Berger [1], has Fitting length at most 2 . 
(ii) The proof given for Lemma 3.2 of [14] suffices to establish (ii), since the solubility of $G$ removes the need to employ the Thompson normal $p$-complement theorem in that proof.

(iii) See Theorem 3.3(i) of Ralston [14].

We shall rely upon the ensuing four results frequently.

(2.11) (see (2.19) of [16]). Let $G$ be a finite soluble group admitting a coprime automorphism $\alpha$ of prime order. Suppose $G=M N \geqq N$, where $M$ and $N$ are $\alpha$-invariant subgroup of $G$ with $(|M|,|N|)=1$. If $M$ centralizes $C_{N}(\alpha)$, then $[[M, \alpha], N]=1$ unless $2 \in \pi(M)$ and $|\langle\alpha\rangle|$ is a Fermat prime.

(2.12) (see (2.20) of [16]). Assume $G, M, N$ and $\alpha$ are as given in (2.11). If $M$ centralizes $C_{N}(\alpha)$ and $M$ is abelian, then $[M, \alpha]$ centralizes $N$.

(2.13) Let $G$ be a soluble group admitting a coprime automorphism $\alpha$ of prime order and let $H$ be an $\alpha$-invariant Hall $\pi$ subgroup of $G$. For (i) and (ii) assume that $2 \notin \pi(H)$ or $|\langle\alpha\rangle|$ is a non-Fermat prime. If $C_{G}(\alpha)$ is a $\pi$-group, then

(i ) $H=O_{\pi}(G) C_{H}(\alpha)$;

(ii) $G=O_{\pi, \pi^{\prime}, \pi}(G)$ (that is, $G$ has $\pi^{\prime}$-length at most one); and

(iii) for $J$ an $\alpha$-invariant abelian $\pi$-group, $J=C_{J}(\alpha)\left(J \cap O_{\pi}(G)\right)$.

Proof. (i) See (2.21) of [16].

(ii) By (2.3)(xiv) $G$ will have an $\alpha$-invariant Hall $\pi^{\prime}$-subgroup, call it $K$. Set $\bar{G}=G / O_{\pi}(G)$, and use bars to denote images of subgroups of $G$ in $\bar{G}$. Using (i) and (2.3) (iii) gives $\bar{H}=C_{\bar{H}}(\alpha)$. By hypothesis, $\bar{K}=[\bar{K}, \alpha]$ and so $\bar{K} \leqq \bar{G}$ (by (2.3)(ix)). This establishes (ii).

(iii) Using (2.12) in the proof of $[(2.21) ; 16]$ gives (iii).

(2.14) Let $G$ be a soluble group which admits an automorphism $\alpha$ of square-free order. Suppose $G=J H \geqq H$, where $J$ and $H$ are $\alpha$-invariant subgroups of coprime order, $H$ is nilpotent and $J$ normalizes an $\alpha$-invariant subgroup, $K$, of $H$.

(i) Suppose that $|\langle\alpha\rangle|$ is a prime number, $C_{H}(\alpha) \leqq K$ and $(|G|,|\langle\alpha\rangle|)=1$. Further, assume at least one of the following holds: $2 \notin \pi(J) ;|\langle\alpha\rangle|$ is a non-Fermat prime; or $J$ is abelian. Then $[[J, \alpha], H] \leqq K$ (or, equivalently, $H=K C_{H}([J, \alpha])$ ).

(ii) If $\alpha$ acts fixed-point-freely upon $J,(|H|,|\langle\alpha\rangle|)=1$ and $C_{H}\left(\alpha^{j}\right) \leqq K$ for all $j, 1 \leqq j\langle|\langle\alpha\rangle|$, then $[J, H] \leqq K$ (or, equivalently, $\left.H=K C_{H}(J)\right)$. 
Proof. Constructing a 'normalizer chain' between $K$ and $H$ (as in, for example, (2.26) of [16]) and then using either (2.11) and (2.12) (for part (i)) or (2.8) (for part (ii)) together with (2.3)(ii) yields (2.14).

In $\S 4$ we shall have recourse to the following theorem.

(2.15) (see [Satz 17.13; 9]). Let $P$ be an extra-special $p$-group of order $p^{2 m+1}$ admitting a coprime cyclic automorphism group $A$ which centralizes $Z(P)$ and acts regularly upon $P / Z(P)$. Let $G$ denote the semi-direct product of $P$ with $A$. Suppose $G$ is faithfully and irreducibly represented on the $K$-vector space $V$, where $K$ is an algebraically closed field and $(\operatorname{char} K,|G|)=1$. If $\chi$ denotes the character of this representation of $G$ on $V$ and $\rho$ the regular character of $A$, then

$$
\left.\chi\right|_{A}=\frac{\left(p^{m}-\delta\right) \rho}{|A|}+\delta \mu
$$

where $\mu$ is some irreducible character of $A$ and $\delta=+1$ or -1 .

(2.16) (see $[(2.25) ; 16])$. Let $G$ be a soluble group admitting the automorphism $\alpha$ fixed-point-freely. Suppose $H$ is an $\alpha$-invariant nilpotent Hall $\pi$-subgroup of $G$ which contains an $\alpha$-invariant subgroup $J$ such that $C_{H}(J) \leqq J$. If, further, $2 \notin \pi$, then every $\pi^{\prime}$ subgroup of $G$ which is normalized by $J$ is contained in $O_{\pi^{\prime}}(G)$.

A further piece of notation is the following: if $G$ is a group which admits $A$ as an operator group, then, for $a \in A, C_{G}(a)$ will frequently be denoted by $G_{a}$.

As intimated in the introduction, most of our subsequent arguments will be in the context of certain 'minimal situations'. Accordingly, we introduce the following hypotheses.

The pair $(G, A)$ will be said to satisfy Hypothesis I if $G$ is a nonsoluble group admitting $A$ as an automorphism group fixed-pointfreely and satisfying

(i) if $A$ is noncyclic, then $(|G|,|A|)=1$;

(ii) all proper $A$-invariant subgroups of $G$ are soluble;

(iii) $G$ possesses no nontrivial proper $A$-invariant normal subgroups; and

(iv) if $B$ is a proper subgroup of $A$ and $H$ is a $B$-invariant subgroup of $G$ upon which $B$ acts fixed-point-freely, then $H$ is soluble.

The pair $(G, A)$ will be said to satisfy Hypothesis II if

(i) $(G, A)$ satisfies Hypothesis I with $A$ cyclic of square-free order. 
Finally, the pair $(G, A)$ will be said to satisfy Hypothesis III if it satisfies Hypothesis II and, additionally,

(i) $|A|=r s t(r, s$ and $t$ being primes);

(ii) $(|G|,|A|)=1$; and

(iii) $|A|$ is odd.

Part of the argument presented in the proof of $[(2.28) ; 16]$ shows

(2.17) If $G$ satisfies Hypothesis I, then $G$ is a non-abelian simple group.

Remarks. (i) Part (iv) of Hypothesis I will be satisfied provided that the 'fixed-point-free automorphism conjecture' has been verified for all $B \supsetneqq A$.

(ii) In Hypothesis III we note that part (iii) is a consequence of part (ii) (of Hypothesis III), the nonsolubility of $G$ and a theorem due to Feit and Thompson [4].

As in [16], when $(G, A)$ satisfies Hypothesis $I$ and $H$ is a proper $A$-invariant subgroup of $G,(H)_{\pi}$ will denote the (unique) $A$-invariant Hall $\pi$-subgroup of $H$ (which exists by $(2.1)(\mathrm{v})$ ), where $\pi$ is a set of primes.

For the remainder of this section we shall assume $(G, A)$ to be a pair which satisfies Hypothesis I. Additionally, we will suppose (up to and including (2.23) that $G$ possesses $A$-invariant nilpotent Hall $\lambda$ - and $\mu$-subgroups denoted (respectively) by $L$ and $M$. In such a situation, $\mathscr{C}(\lambda, \mu)$ is defined to be the set of all maximal $A$ invariant $\{\lambda \cup \mu\}$-subgroups of $G$. The 'local analysis' of $G$ is concerned with the number of elements of $\mathscr{C}(\lambda, \mu)$ and their group theoretic structure. Clearly, (using (2.1)) $|\mathscr{M}(\lambda, \mu)|=1$ if and only if $L M=M L$. Because $\lambda \cap \mu \neq \varnothing$ forces $L M=M L$, we further suppose, in the 'local analysis', that $\lambda \cap \mu=\varnothing$. Further details of the methods adopted in this type of work may be found in $\S 2$ of [16]; (note that using our (2.7) in place of $[(2.12) ; 16]$ in $\S 2$ of [16] removes the need for coprimeness when $A$ is cyclic).

The next three results are some of the tools used in the 'local analysis' mentioned above.

(2.18) (Martineau [11]; see also $[(2.10), 16])$. Let $H \in \mathscr{C}(\lambda, \mu)$ and let $J$ be an $A$-invariant $\{\lambda \cup \mu\}$-subgroup of $G$. If $J \cap O_{\lambda}(H) \neq$ $1 \neq J \cap O_{k}(H)$, then $J \leqq H$. 
(2.19) (Martineau [11] and Ralston [14]; see also $[(2.11) ; 16])$. Suppose $H \in \mathscr{M}(\lambda, \mu)$ with $O_{\mu}(H) \neq 1 \neq O_{\lambda}(H)$ and suppose $J$ is a nontrivial $A$-invariant subgroup of $F(H)$. Then $\left(N_{G}(J)\right)_{\{\lambda \cup \mu\}} \leqq H$.

Let $X$ (respectively $Y$ ) denote the largest $A$-invariant subgroup of $L$ (respectively $M$ ) which permutes with $M$ (respectively $L$ ). Note that $\{L Y, M X\} \subseteq \mathscr{M}(\lambda, \mu)$.

(2.20) (Martineau [12] and Pettet [13]; see also [(2.18); 16]). $L=O_{\lambda}(L Y) X$ and $M=O_{i k}(M X) Y$.

(2.21) (i ) $L Y \cap M X=X Y$.

(ii) $O_{\lambda}(X Y)=O_{\lambda}(L Y) \cap X$ and $O_{\mu}(X Y)=O_{\mu}(M X) \cap Y$.

(iii) If $O_{\mu}(L Y) \neq 1 \neq O_{\lambda}(M X)$, then $M L=L M$.

(iv) If $O_{\mu}(L Y) \neq 1$, then $C_{M}(Y) \leqq Y$ and, in particular, $Z(M) \leqq Y$.

(v) If $Y \leqq L Y$, then either $L M=M L$ or $Y=1$.

(vi) If $L=X C_{L}(Y)$ and $\mathscr{C}(\lambda, \mu)=\{L Y, M X\}$, then either $L M=M L$ or $Y=1$.

Proofs. Proofs for (i), (ii) and (iii) are given in $[(2.17) ; 16]$. The remaining assertions are well known (and straightforward). Part (iv) follows from the fact that $\left(N_{G}\left(O_{\mu}(L Y)\right)\right)_{\{\lambda \cup \mu\}}=L Y$ and parts (v) and (vi) by using the nilpotence of $M$.

Concerning the number of elements possessed by $\mathscr{C}(\lambda, \mu)$ we have

(2.22) (Rowley [Lemma $3.3 ; 16]$ ). If $A$ is an abelian group of square-free exponent and $(|G|,|A|)=1$, then $|\mathscr{L}(\lambda, \mu)| \leqq 2$.

(2.23) (Rowley [Lemma 4.2; 16]). Suppose $(|G|,|A|)=1, L M \neq$ $M L, 2 \notin \mu$ and $C_{L}(\alpha) \leqq X$ where $|\langle\alpha\rangle|$ is a prime and $\alpha \in Z(A)^{\sharp}$. Then either $Y \leqq C_{M}(\alpha)$ or $O_{\mu}(L Y) \neq 1$.

In certain situations that we shall encounter, the following result is indispensable.

(2.24) (Rowley). Assume that $(|G|,|A|)=1$. If $B$ is a subgroup of $A$ for which $C_{G}(B)$ is soluble, then $C_{G}(B)$ does not contain a Sylow 2-subgroup of $G$.

Proof. If $C_{G}(B)$ were to contain a Sylow 2-subgroup of $G$, then, by $[(2.28) ; 16],(G, A)$ would not satisfy Hypothesis I. Thus (2.24) holds. 
Let $L$ and $M$ be two proper $A$-invariant subgroups of $G$ (not necessarily nilpotent Hall subgroups). We define $\mathscr{P}_{L}(M)$ to be the largest $A$-invariant subgroup of $L$ which is permutable with $M$.

(2.25) (i ) $\mathscr{P}_{L}(M)=\left\langle L_{1}\right| L_{1}$ is an $A$-invariant subgroup of $L$ permutable with $M\rangle$.

(ii) $\mathscr{P}_{L}(M)=\left\langle L_{1}\right| L_{1}$ is a subgroup of $L$ permutable with $\left.M\right\rangle$.

Proof. (i) This is immediate from the definition of $\mathscr{P}_{L}(M)$.

(ii) Let $\mathscr{P}_{L}^{\prime}(M)$ denote the right hand side of the equation in (ii). Clearly, from (i), $\mathscr{P}_{L}(M) \leqq \mathscr{P}_{L}^{\prime}(M)$. Since $L$ and $M$ are both $A$-invariant, we observe that $\mathscr{P}_{L}^{\prime}(M)$ is $A$-invariant and plainly permutes with $L$. Thus $\mathscr{P}_{L}^{\prime}(M) \leqq \mathscr{P}_{L}(M)$, and (ii) is proven.

The penultimate result of this section plays a vital role in manufacturing (interesting) subgroups of $G$.

(2.26) (Rowley $[(2.29) ; 16])$. Suppose $G$ possesses an $A$-invariant Hall $\pi$-subgroup, $H$ say, and let $L$ and $M$ be proper $A$-invariant subgroups of $G$ with $(|L|,|M|)=1$. Then either $M \mathscr{P}_{L}(M)=G$ or the $A$-invariant Hall $\pi$-subgroup of $\mathscr{P}_{L}(M)$ is $\mathscr{P}_{I I \cap L}(M)$.

(2.27) Suppose $G$ is a group and let $L$ and $M$ be subgroups of $G$ with $L \cap M=1$. If $M_{1}, \cdots, M_{f}$ are subgroups of $M$ each of which permutes with $L$, then $L$ permutes with $\bigcap_{i=1}^{f} M_{i}$.

Proof. Suppose $f=2$, and let $l \in L$ and $m \in M_{1} \cap M_{2}$. Then $m_{1} l_{1}=l m=m_{2} l_{2}$ for some $l_{1}, l_{2} \in L$ and some $m_{\imath} \in M_{i}(i=1,2)$. So $m_{2}^{-1} m_{1}=l_{2} l_{1}^{-1} \in M \cap L=1$. Hence $l_{1}=l_{2}$ and $m_{1}=m_{2} \in M_{1} \cap M_{2}$. Therefore $L$ and $M_{1} \cap M_{2}$ permute. An easy induction now completes the proof of the lemma.

3. The 'Star-subgroup'. We begin by giving the definition of the 'star-subgroup'.

Definition 3.1. Suppose $G$ is a finite group admitting the automorphism $\alpha$. Let $H$ be an $\alpha$-invariant subgroup of $G$ and let $\langle\beta\rangle$ be a subgroup of $\langle\alpha\rangle$. Then set $H_{\langle\beta\rangle}^{*}=\left\langle C_{H}\left(\beta^{j}\right)\right| 1 \leqq j\langle|\langle\beta\rangle|\rangle$ ("the star-subgroup of $H$ with respect to $\langle\beta\rangle$ ).

That in, $H_{\langle\beta\rangle}^{*}$ is defined to be the subgroup of $H$ which is generated by the fixed-point sets (in $H$ ) of the nontrivial powers of $\beta$. When $\langle\beta\rangle=\langle\alpha\rangle$ and there is no possibility of confusion, $H_{\langle\alpha\rangle}^{*}$ will be written as just $H^{*}$. 
REMARK. We may rephrase (2.8) as follows:- suppose $G$ is a finite group admitting a fixed-point-free automorphism $\alpha$ of squarefree order and let $P$ denote the $\alpha$-invariant Sylow $p$-subgroup of $G$. If $P^{*}=1$, then $P$ is a direct summand of $G$.

In Lemma 3.3, after a further definition, we collect together some elementary properties of the 'star-subgroup'.

DEFINITION 3.2. Let $G$ be a group admitting a fixed-point-free automorphism $\alpha$, let $H$ be an $\alpha$-invariant subgroup of $G$ and let $\beta \in\langle\alpha\rangle$. Then $H$ is said to be star-covered with respect to $\langle\beta\rangle$ if only if for each $\alpha$-invariant subgroup $K$ of $H, K=K_{\langle\beta\rangle}^{*}$.

Again, when $\langle\beta\rangle=\langle\alpha\rangle$, and confusion is unlikely, $H$ will just be referred to as being star-covered. Observe that, if $H$ is an $\alpha$ invariant subgroup of $G$ which is star-covered with respect to $\langle\beta\rangle$, then all $\alpha$-invariant subgroups of $H$ are, likewise, star-covered with respect to $\langle\beta\rangle$.

Lemma 3.3. Let $G, \alpha, H$ and $\beta$ be as defined in Definition 3.1.

(i) $H_{\langle\beta\rangle}^{*}$ is an $\alpha$-invariant subgroup of $G$.

(ii) If $K$ is an $\alpha$-invariant subgroup of $H$, then $K_{\langle\beta\rangle}^{*} \leqq H_{\langle\beta\rangle}^{*}$.

(iii) If $\langle\gamma\rangle \leqq\langle\beta\rangle$, then $H_{\langle\gamma\rangle}^{*} \leqq H_{\langle\beta\rangle}^{*}$.

(iv) $H_{\langle\beta\rangle}^{*}=\left\langle C_{H}(\gamma) \mid \gamma \in \Omega_{1}(\langle\beta\rangle)^{\#}\right\rangle$.

(v) Suppose $\Omega_{1}(\langle\beta\rangle)$ is of order $r_{1}, \cdots, r_{m}$ (where $r_{i}$ is a prime, $1 \leqq i \leqq m)$ and for $j=1, \cdots, m$, let $\beta_{j}$ denote an element of $\Omega_{1}(\langle\beta\rangle)$ of order $r_{j}$. Then $H_{\langle\beta\rangle}^{*}=\left\langle C_{H}\left(\beta_{j}\right) \mid j=1, \cdots, m\right\rangle$.

(vi) If $(|H|,|\langle\beta\rangle|)=1$ and $N$ is an $\alpha$-invariant normal subgroup of $H$, then $(H / N)_{\langle\beta\rangle}^{*}=\left(H_{\langle\beta\rangle}^{*} N\right) / N$.

(vii) Suppose that $(|H|,|\langle\beta\rangle|)=1$ and that $N$ is an $\alpha$-invariant normal subgroup of $H$. If both $H / N$ and $N$ are star-covered with respect to $\langle\beta\rangle$ then $H$ is star-covered with respect to $\langle\beta\rangle$.

Proof. Parts (i), (ii) and (iii) follow from the definition of the star subgroup.

(iv) Since, for each $j\left(1 \leqq j\langle|\langle\beta\rangle|),\left\langle\beta^{j}\right\rangle \cap \Omega_{1}(\langle\beta\rangle) \neq 1, H_{\langle\beta\rangle}^{*} \leqq\right.$ $\left\langle C_{H}(\gamma) \mid \gamma \in \Omega_{1}(\langle\beta\rangle)^{\sharp}\right\rangle\left(\leqq H_{\langle\beta\rangle}^{*}\right)$ whence (iv) follows.

(v) Let $\gamma \in \Omega_{1}(\langle\beta\rangle)^{\sharp}$. Then $\left\langle\beta_{k}\right\rangle \leqq\langle\gamma\rangle$ for some $k \in\{1, \cdots, m\}$. Now (v) follows from (iv).

(vi) Let bars denote images in $H / N$. As $(|H|,|\langle\beta\rangle|)=1$, by (2.3)(iii), $\overline{C_{H}\left(\beta^{j}\right)}=C_{\bar{H}}\left(\beta^{j}\right)$ for all $j, 1 \leqq j\langle|\langle\beta\rangle|$, and so it follows that

$$
\begin{aligned}
(\bar{H})_{\langle\beta\rangle}^{*} & =\left\langle C_{\bar{H}}\left(\beta^{j}\right)|1 \leqq j<|\langle\beta\rangle \mid\right\rangle \\
& =\left\langle\overline{C_{H}\left(\beta^{j}\right)}|1 \leqq j<|\langle\beta\rangle \mid\right\rangle \\
& =\left\langle\overline{C_{H}\left(\beta^{j}\right)|1 \leqq j<|\langle\beta\rangle|\rangle}\right. \\
& =\overline{\left(H_{\langle\beta\rangle}^{*}\right)} .
\end{aligned}
$$


(vii) Let $K$ be an $\alpha$-invariant subgroup of $H$. Since $H$ is starcovered with respect to $\langle\beta\rangle,(K \cap N)_{\langle\beta\rangle}^{*}=K \cap N$. Now $K N / N \cong$ $K / K \cap N$ (and this isomorphism commutes with the $\alpha$ action). Thus, since $H / N$ is star-covered with respect to $\langle\beta\rangle,(K / K \cap N)_{\langle\beta\rangle}^{*}=K / K \cap N$. By (vi) $(K / K \cap N)_{\langle\beta\rangle}^{*}=\left(\left(K_{\langle\beta\rangle}^{*}\right)(K \cap N)\right) / K \cap N$ and so $K=K_{\langle\beta\rangle}^{*}$ (because $\left.K_{\langle\beta\rangle}^{*} \geqq K \cap N\right)$. Therefore $H$ is star-covered with respect to $\langle\beta\rangle$.

For Lemma 3.4 and its corollary, and Lemmas 3.6 and 3.7, $(G, A)$ will be assumed to be a pair which satisfies Hypothesis I with $A(=\langle\alpha\rangle)$ cyclic and, furthermore, the following additional properties are assumed to hold:-

(i) $\pi$ is a subset of $\pi(G)$ for which $\alpha^{k}=\beta$ acts fixed-pointfreely upon all $\alpha$-invariant $\pi$-subgroups of $G$ (that is, $C_{G}(\beta)$ is a $\pi^{\prime}$ subgroup); and

(ii) $p \in \pi$ with $(p,|\langle\beta\rangle|)=1$. Also, $P$ will be used to denote the $\alpha$-invariant Sylow $p$-subgroup of G.

Lemma 3.4. If $R$ is a nontrivial $\alpha$-invariant p-subgroup of $G$ containing $P_{\langle\beta\rangle}^{*}$, then $\left(N_{G}(R)\right)_{\pi} \leqq\left(N_{G}(P)\right)_{\pi}$.

Proof. Choose $\widetilde{R}$ maximal with respect to the following properties.

(1) $\widetilde{R}$ is an $\alpha$-invariant $p$-subgroup;

(2) $R \leqq \widetilde{R}$; and

(3) $\left(N_{G}(R)\right)_{\pi} \leqq\left(N_{G}(\widetilde{R})\right)_{\pi}$.

Clearly, there exists at least one such $\widetilde{R}$.

Since $\widetilde{R}$ is nontrivial, because Hypothesis I holds for $(G, A)$, $N_{G}(\widetilde{R})$ is a proper $\alpha$-invariant subgroup of $G$ and therefore must be soluble. Hence $K / \widetilde{R}$ is soluble, where $K=\left(N_{G}(\widetilde{R})\right)_{\pi}$.

As $(p,|\langle\beta\rangle|)=1,\left(N_{P}(\widetilde{R}) / \widetilde{R}\right)_{\langle\beta\rangle}^{*}=1$ by Lemma 3.3 (vi) and so, from (2.9), $\quad N_{P}(\widetilde{R}) / \widetilde{R} \leqq K / \widetilde{R}$ since $K$ admits $\beta$ fixed-point-freely. Thus $N_{P}(\widetilde{R}) \leqq K$ which, together with (3), implies that

$$
\left(N_{G}(R)\right)_{\pi} \leqq\left(N_{G}(\widetilde{R})\right)_{\pi}=K \leqq\left(N_{G}\left(N_{P}(\widetilde{R})\right)\right)_{\pi} .
$$

Since $N_{P}(\widetilde{R})$ also satisfies $(1)$ and $(2), N_{P}(\widetilde{R})=\widetilde{R}$ by the maximal choice of $\widetilde{R}$. Consequently $P=\widetilde{R}$ and therefore $\left(N_{G}(R)\right)_{\pi} \leqq\left(N_{G}(P)\right)_{\pi}$.

We have the immediate

COROLlaRY 3.5. If $R$ is a nontrivial $\alpha$-invariant p-subgroup of $G$ containing $P_{\langle\beta\rangle}^{*}$ with $\langle\beta\rangle=\langle\alpha\rangle$, then $N_{G}(R) \leqq N_{G}(P)$.

Lemma 3.6. Suppose $A(=\langle\alpha\rangle)$ is of square-free order and let 
$R$ be a nontrivial $\alpha$-invariant $p$-subgroup containing $P_{\langle\beta\rangle}^{*}$. Then, setting $K=N_{G}(R)_{\pi \backslash \backslash p\rangle}$, of $K$;

(i) $[P, L]=[R, L] \leqq R$, where $L$ is any $\alpha$-invariant subgroup

(ii) $C_{K}\left(P_{\langle\beta\rangle}^{*}\right)=C_{K}(P)$; and

(iii) $\left(C_{G}\left(P^{*}\right)\right)_{p^{\prime}}=\left(C_{G}(P)\right)_{p^{\prime}}$ (recall that $P^{*}$ means $\left.P_{\langle\alpha\rangle}^{*}\right)$.

Proof. (i ) From Lemma 3.4, $L \leqq\left(N_{G}(P)\right)_{\pi} \leqq N_{G}(P)$. Now $\beta$ acts fixed-point-freely upon $L$ and $P_{\langle\beta\rangle}^{*} \leqq R$, and so applying (2.14)(ii) to $P L$, yields that $[P, L] \leqq R$. By $(2.3)$ (vii) (with $L$ as a coprime operator group on $P)[P, L]=[R, L]$.

(ii) If $P_{\langle\beta\rangle}^{*}=1$, then the result is obvious from (2.8). So we may suppose that $P_{\langle\beta\rangle}^{*} \neq 1$. Applying part (i) with $R=P_{\langle\beta\rangle}^{*}$ and $L=C_{K}\left(P_{\langle\beta\rangle}^{*}\right)$ yields that

$$
\left[P, C_{K}\left(P_{\langle\beta\rangle}^{*}\right)\right]=\left[P_{\langle\beta\rangle}^{*}, C_{K}\left(P_{\langle\beta\rangle}^{*}\right)\right]=1
$$

which, together with $C_{K}(P) \leqq C_{K}\left(P_{\langle\beta\rangle}^{*}\right)$, gives $C_{K}(P)=C_{K}\left(P_{\langle\beta\rangle}^{*}\right)$.

(iii) This follows from (ii) with $\pi=\pi(G)$ (since $\alpha$ acts fixedpoint-freely on $G$ ).

We note the following

LEMMA 3.7. If $L$ is an $\alpha$-invariant nilpotent Hall $\lambda$-subgroup of $G$ with $\lambda \subseteq \pi$, then analogues of Lemmas 3.4 and 3.6 holds.

Lemma 3.8. Let $(G, A)$ be a pair satisfying Hypothesis I with $A(=\langle\alpha\rangle)$ cyclic. Moreover, suppose that $|A|$ is square-free and that $H$ is an $\alpha$-invariant subgroup of $G$ upon which $\beta(\in A)$ acts fixedpoint-freely. Let $P$ denote the $\alpha$-invariant Sylow p-subgroup of $H$ and set $M=O_{p^{\prime}}(H)$. If $(p,|\langle\beta\rangle|)=1$, then $[P, M]=\left[P_{\langle\beta\rangle}^{*}, M\right]$.

Proof. Since $\left[P_{\langle\beta\rangle}^{*}, M\right] \leqq[P, M]$, it will be sufficient to establish the reverse inclusion. Let $R$ be an $\alpha$-invariant $p$-subgroup which is maximal (under inclusion) subject to containing $P_{\langle\beta\rangle}^{*}$ and satisfying $[R, M] \leqq\left[P_{\langle\beta\rangle}^{*}, M\right]$. From (2.3)(i) $M=C_{M}(R)[R, M]$. Now, from Lemma $3.6(\mathrm{ii}), C_{M}\left(N_{P}(R)\right)=C_{M}\left(P_{\langle\beta\rangle}^{*}\right)=C_{M}(R)$ since $N_{P}(R) \geqq R \geqq P_{\langle\beta\rangle}^{*}$. So $M=C_{M}(R)[R, M]=C_{M}\left(N_{P}(R)\right)[R, M]$. Because $[R, M]$ is $N_{P}(R)$ invariant by $(2.3)(\mathrm{vi})$, employing $(2.3)(\mathrm{ix})$ yields that $\left[N_{P}(R), M\right] \leqq$ $[R, M]$. Hence $\left[N_{P}(R), M\right] \leqq\left[P_{\langle\beta\rangle}^{*}, M\right]$ and so, by the choice of $R$, $N_{P}(R)=R$. Therefore $P=R$, and the lemma follows.

LEMMA 3.9. Suppose $G$ is a soluble group admitting the automorphism $\alpha$ fixed-point-freely and suppose $|\langle\alpha\rangle|$ is square-free. Let $P$ denote the $\alpha$-invariant Sylow p-subgroup of $G$ and assume 
$(p,|\langle\alpha\rangle|)=1$. If $K$ is an $\alpha$-invariant $p^{\prime}$-subgroup of $G$ normalized by $P^{*}$, then $K \leqq O_{p^{\prime}}(G)$.

Proof. Set $\bar{G}=G / O_{p^{\prime}}(G)$ and use the usual 'bar notation'. By Lemma $3.3(\mathrm{vi}),(\bar{P})^{*}=\left(\bar{P}^{*}\right)$ (since $(p,|\langle\alpha\rangle|=1)$. Hence

$$
\left[\left(O_{p}(\bar{G})\right)^{*}, \bar{K}\right] \leqq O_{P}(\bar{G}) \cap \bar{K}=1 .
$$

Since $\bar{K}$ normalizes $\left(O_{p}(\bar{G})\right)^{*}$, applying $(2.14)\left(\right.$ ii) to $O_{p}(\bar{G}) \bar{K}$ yields that $\left[O_{p}(\bar{G}), \bar{K}\right] \leqq\left(O_{p}(\bar{G})\right)^{*}$ and hence $\left[O_{p}(\bar{G}), \bar{K}\right]=\left[\left[O_{p}(\bar{G}), \bar{K}\right], \bar{K}\right]=1$. Now $C_{\bar{G}}\left(O_{p}(\bar{G})\right) \leqq O_{p}(\bar{G})$ by a result of Hall and Higman (see Theorem 6.3.2. of [6]) and hence $\bar{K}=1$. Thus $K \leqq O_{p^{\prime}}(G)$.

Suppose, for the remainder of this section, that $G$ is a group admitting a fixed-point-free automorphism $\alpha$ of square-free order $\gamma_{1} \cdots r_{n}$ (where $r_{i}$ is a prime, $\left.i=1, \cdots, n\right)$. Set $\Lambda=\{1, \cdots, n\}$.

For each $i \in \Lambda, \alpha_{\imath}$ will denote a generator of the Sylow $r_{\imath}$-subgroup of $\langle\alpha\rangle$ (so $\alpha_{i}^{r_{i}}=1$ ).

We now make a further definition.

Definition 3.10. Let $P$ be an $\alpha$-invariant Sylow $p$-subgroup of $G$ where $p \in \pi(G)$. Then $P$ is said to be of type $\Gamma$ (where $\Gamma$ is a subset of $\Lambda$ ) if and only if $i \in \Gamma$ implies $P_{\alpha_{i}} \neq 1$ and $i \notin \Gamma$ implies $P_{\alpha_{i}}=1$ (recall that $P_{\alpha_{i}}$ stands for $C_{P}\left(\alpha_{i}\right)$ ).

REMARKs. (i) If $P$ is an $\alpha$-invariant Sylow $p$-subgroup of $G$ of type $\Gamma$ (where $I \cong \Lambda$ ), then $P^{*}=\left\langle P_{\alpha_{i}} \mid i \in \Gamma\right\rangle$.

(ii) If $(G,\langle\alpha\rangle)$ also satisfies Hypothesis I, then (2.8) shows that $G$ can have at most $2^{n}-1$ possible types of $\alpha$-invariant Sylow subgroups.

LEMMA 3.11. Let $P$ and $Q$ denote (respectively) the $\alpha$-invariant Sylow $p$ - and $q$-subgroups of $G$ with $p \neq q$. Suppose $P$ is of type $\Gamma$ (where $\Gamma \leqq \Lambda)$ and set $\gamma=\prod_{i \in \Gamma} \alpha_{\imath}$ (for $\Gamma=\varnothing$ we make the convention $\left.\gamma=\prod_{i \in \dot{b}} \alpha_{i}=1 \in\langle\alpha\rangle\right)$. Then $\left[P, C_{Q}(\gamma)\right]=1$.

Proof. This will be done by induction on $|\langle\alpha\rangle|$. First observe, by $(2.2)$ (i) and (2.8), that we may assume $n>1$ and $\Gamma \neq \phi$.

Let $j \in \Gamma$. Since $C_{G}\left(\alpha_{j}\right)$ admits $\alpha_{1} \cdots \alpha_{j-1} \alpha_{j+1} \cdots \alpha_{n}=\beta$ fixedpoint-freely and $|\langle\beta\rangle|\left\langle|\langle\alpha\rangle|\right.$, we deduce that $C_{G}\left(\alpha_{j}\right)$ satisfies the conclusion of the lemma. Now (by (2.1)(iii)) $C_{P}\left(\alpha_{j}\right)$ is the $\alpha$-invariant Sylow $p$-subgroup of $C_{G}\left(\alpha_{j}\right)$ and hence must also be the (unique) $\beta$ invariant Sylow $p$-subgroup of $C_{G}\left(\alpha_{j}\right)$ (see (2.1)(ii)). Likewise $C_{Q}\left(\alpha_{j}\right)$ is the $\beta$-invariant Sylow $q$-subgroup of $C_{G}\left(\alpha_{j}\right)$. With respect to $\beta$, $C_{P}\left(\alpha_{3}\right)$ will be of type $\Delta$ where $\Delta \subseteq \Gamma \backslash\{j\}$. Set $\delta=\prod_{i \in \Delta} \alpha_{i}$. Then we 


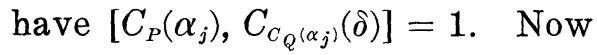

$$
\begin{aligned}
C_{C_{Q^{\left(\alpha_{j}\right)}}}(\delta) & =C_{Q}\left(\alpha_{j}\right) \cap C_{G}(\delta) \\
& =C_{Q}\left(\alpha_{j}\right) \cap\left(\bigcap_{i \in \Delta} C_{G}\left(\alpha_{i}\right)\right) \\
& =Q \cap\left(\bigcap_{i \in \Delta \cup\{j\}} C_{G}\left(\alpha_{i}\right)\right) \\
& \geqq Q \cap\left(\bigcap_{i \in \Gamma} C_{G}\left(\alpha_{i}\right)\right)=Q \cap C_{G}(\gamma)=C_{Q}(\gamma) .
\end{aligned}
$$

Thus, for each $j \in \Gamma,\left[C_{P}\left(\alpha_{j}\right), C_{Q}(\gamma)\right]=1$. Consequently $\left[P^{*}, C_{Q}(\gamma)\right]=1$.

Choose $D$ to be a maximal (under set theoretic inclusion) $\alpha$ invariant subgroup of $P$ containing $P^{*}$ which centralizes $C_{Q}(\gamma)$. Set $N=N_{G}(D), \bar{N}=N / D$ and use the 'bar notation'. If $(p,|\langle\alpha\rangle|)=1$, then, since $P^{*} \leqq D, \overline{N_{P}(D)^{*}}=1$ by Lemma 3.3 (vi). Thus $\overline{N_{P}(D)}$ is a direct summand of $\bar{N}$ by $(2.8)$ whence $1=\left[\left[N_{P}(D), C_{Q}(\gamma)\right], C_{Q}(\gamma)\right]=$ $\left[N_{P}(D), C_{Q}(\gamma)\right]$. Therefore $N_{P}(D)=D$ and hence $P=D$. This shows that $\left[P, C_{Q}(\gamma)\right]=1$ when $(p,|\langle\alpha\rangle|)=1$. Now suppose that $(p,|\langle\alpha\rangle|) \neq 1$ and, for convenience, we assume $p=r_{1}$. Observe that $\left(\left(\overline{N_{P}(D)}\right)_{\alpha_{1}}\right)_{\left\langle\alpha_{2} \ldots \alpha_{n}\right\rangle}^{*}=1$ and so $\left.\left(\overline{N_{P}(D}\right)\right)_{\alpha_{\perp}}$ is a direct summand of $(\bar{N})_{\alpha_{1}}$ by (2.8). From a well-known property of p-groups, $1 \in \Gamma$ and so $\overline{C_{Q}(\gamma)} \leqq(\bar{N})_{\alpha_{1}}$. Hence, if $E$ denotes the inverse image of $\overline{\left(N_{P}(D)\right)_{\alpha_{1}}}$ in $N_{P}(D),\left[E, C_{Q}(\gamma)\right]=\left[\left[E, C_{Q}(\gamma)\right], C_{Q}(\gamma)\right]=1$. Therefore $E=D$ and so we conclude that $\overline{N_{P}(D)}=1$. Consequently $P=D$ and so $\left[P, C_{Q}(\gamma)\right]=1$ in this case as well.

Definition 3.12. Let $\Gamma$ be a subset of $\Lambda$. Then define $L_{c}=\langle P| P$ is an $\alpha$-invariant Sylow subgroup of $G$ of type $\left.(\Lambda \backslash \Gamma)\right\rangle$; and

$$
\left.\mathscr{L}_{\Gamma}=\left\langle L_{\Delta}\right| \text { all subsets } \Delta \text { of } \Lambda \text { with } \Delta \supseteqq \Gamma\right\rangle \text {. }
$$

Set $\pi_{\Gamma}=\pi\left(L_{\Gamma}\right)$ and $\hat{\pi}_{\Gamma}=\pi\left(\mathscr{L}_{\Gamma}\right)$.

For $i \in \Lambda$ we shall write $\mathscr{L}_{\{i\}}, L_{\{i\}}, \pi_{\{i\}}$ and $\hat{\pi}_{\{i\}}$ as (respectively) $\mathscr{L}_{i}, L_{i}, \pi_{i}$ and $\hat{\pi}_{i}$, and $L_{\phi}$ will be denoted by $L_{0}$. $i \in \Gamma$.

Remarks. (i) If $\Gamma$ is a subset of $\Lambda$, then $\left(L_{\Gamma}\right)_{\alpha_{i}}=1$ for all

(ii) If $\Gamma$ and $\Delta$ are subsets of $\Lambda$, then $\Delta \cong \Gamma$ implies that $\mathscr{L}_{\Gamma} \leqq \mathscr{L}_{\Delta}$.

Lemma 3.13. Suppose $(G,\langle\alpha\rangle)$ satisfies Hypothesis II and let $\Gamma$ be a subset of $A$. Then

(i) for each $i \in \Lambda, \mathscr{L}_{i}$ is a nilpotent $\alpha$-invariant Hall subgroup of $G$; 
(ii) if $\Gamma \neq \varnothing, Z_{\Gamma}$ is a nilpotent $\alpha$-invariant Hall subgroup of $G$; and

(iii) $\left[\mathscr{C}_{\Gamma},\left(C_{G}(\delta)\right)_{r}\right]=1$ where $\eta=\left(\hat{\pi}_{\Gamma}\right)^{\prime}, \delta=\Pi_{i \in \Delta} \alpha_{i}$ and $\Delta=\Lambda \backslash$.

Proof. (i ) Clearly (because of (2.2)(i)) we may suppose $n>1$ and, without loss of generality, we set $i=1$. Let $P$ and $Q$ be, respectively, (nontrivial) $\alpha$-invariant Sylow $p$ and $q$-subgroups of $G$ such that $P_{\alpha_{1}}=Q_{\alpha_{1}}=1$ with $p \neq q$. Suppose $P$ and $Q$ are, respectively, of type $\Delta$ and $\Gamma$ and set $\delta=\prod_{\imath \in \Delta} \alpha_{i}$ and $\gamma=\prod_{i \in \Gamma}, \alpha_{i}$. Note \lrcorner,$\Gamma \subseteq\{2, \cdots, n\}$.

To establish (i) of the lemma, it will be sufficient to show that $[P, Q]=1$. This may be achieved by demonstrating the existence of an $\alpha$-invariant $\{p, q\}$-subgroup $H_{1}$ for which $P \cap H_{1} \neq 1 \neq Q \cap H_{1}$. Then, if $H$ is a maximal $\alpha$-invariant $\{p, q\}$-subgroup of $G$ containing $H_{1}, H$ is nilpotent since $H_{\alpha_{1}}=1$. As Hypothesis II holds, $N_{G}(P \cap H)$ is soluble. Hence $\left(N_{G}(P \cap H)\right)_{\{p, q\}}=N_{P}(P \cap H) N_{Q}(P \cap H) \geqq H$ and the maximality gives $N_{P}(P \cap H) \leqq H$ which implies $N_{P}(P \cap H)=P \cap H$. Thus $P \leqq H$, and, similarly, $Q \leqq H$ whence $[P, Q]=1$, as desired.

If $\Delta \cap T \neq \varnothing$, then we may take $H_{1}=P_{\alpha_{j}} O_{\alpha_{j}}=\left(C_{G}\left(\alpha_{j}\right)\right)_{p, q)}$ where $j \in \Delta \cap \Gamma$.

So we may suppose that $\Delta \cap \Gamma=\varnothing$. From Lemma 3.11 we have $\left[P,\left(G_{\dot{o}}\right)_{p^{\prime}}\right]=\left[Q,\left(G_{i}\right)_{q^{\prime}}\right]=1$. Since $\Delta \cap \Gamma=\varnothing$, we infer that $C_{G}(\gamma \hat{o})$ is a $\{p, q\}^{\prime}$-subgroup of $G$. Now Hypothesis II requires that $G$ be a nonsoluble group and, since $|\langle\gamma \delta\rangle| \leqq r_{2} \cdots r_{n}\langle|\langle\alpha\rangle|$, again by Hypothesis II (actually Hypothesis I (iv)) $\left(G_{\dot{o}}\right)_{p^{\prime}} \cap\left(G_{i}\right)_{q^{\prime}}=C_{G}(\gamma \delta) \neq 1$. Taking $H_{1}=\left(N_{G}\left(C_{G}(\gamma \delta)\right)\right)_{\{p, q\}}$ in this case, completes the proof of (i).

(ii) Since $\mathscr{L}_{i} \leqq \mathscr{C}_{i}$ for some $i \in A$, (ii) is an immediate consequence of (i).

(iii) If $P$ is the $\alpha$-invariant Sylow $p$-subgroup of $\hat{\mathscr{C}}_{\Gamma}$, then $P$ will be of type $\Psi$ where $\Psi \subseteq \Delta$. From Lemma $3.10\left[P,\left(G_{i}\right)_{p^{\prime}}\right]=1$ where $\gamma=\prod_{\imath \in \Psi} \alpha_{i}$. Since $\Psi \subseteq \Delta, G_{\delta} \leqq G_{\gamma}$ and now (iii) follows.

We next give a result concerning Sylow subgroups of type $\Gamma$ when $n=3$.

Lemma 3.14. Let $P$ and $Q$ be (respectively) the $\alpha$-invariant Sylow $p$ - and $q$-subgroups of $G$ with $p, q \in \pi(G)$ and $p \neq q$. If $P$ and $Q$ are both of type $\Gamma$ and $n=3$, then there exists a nontrivial $\alpha$ invariant p-subgroup $P_{1}$ of $P^{*}$ and a nontrivial $\alpha$-invariant $q$ subgroup $Q_{1}$ of $Q^{*}$ such that $\left[P_{1}, Q_{1}\right]=1$.

Proof. First suppose that at least two of $C_{P}\left(\alpha_{1} \alpha_{2}\right), C_{P}\left(\alpha_{1} \alpha_{3}\right)$ and $C_{P}\left(\alpha_{2} \alpha_{3}\right)$ are nontrivial. Suppose $C_{P}\left(\alpha_{1} \alpha_{2}\right) \neq 1 \neq C_{P}\left(\alpha_{1} \alpha_{3}\right)$. Since $C_{G}\left(\alpha_{1} \alpha_{2}\right)$ and $C_{G}\left(\alpha_{1} \alpha_{3}\right)$ are both nilpotent, either the lemma holds or $C_{Q}\left(\alpha_{1} \alpha_{2}\right)=1=C_{Q}\left(\alpha_{1} \alpha_{3}\right)$. But the latter possibility yields, by (2.8), 
that $\left[C_{P}\left(\alpha_{1}\right), C_{Q}\left(\alpha_{1}\right)\right]=1$. Therefore we may suppose that $C_{P}\left(\alpha_{1} \alpha_{2}\right)=$ $1=C_{P}\left(\alpha_{1} \alpha_{3}\right)$. Again, by $(2.8),\left[C_{P}\left(\alpha_{1}\right), C_{Q}\left(\alpha_{1}\right)\right]=1$, and so the lemma is proved.

REMARK. We note that Lemma 3.14 does not hold for $n \geqq 4$.

In $\S 7$ we shall specialize to the case $|\langle\alpha\rangle|=r_{1} r_{2} r_{3}$; from that section onwards we shall employ the following additional notation.

$$
\begin{array}{llll}
\alpha_{1}=\rho, & \alpha_{2}=\sigma & \text { and } & \alpha_{3}=\tau \\
r_{1}=r, & r_{2}=s & \text { and } & r_{3}=t .
\end{array}
$$

(So $\rho^{r}=\sigma^{s}=\tau^{t}=1$.)

If $i, j \in \Gamma=\{1,2,3\}$ with $i \neq j$, then $L_{\{i, j\}}$ and $\pi_{\{i, j i}$ will be written (respectively) as $L_{i j}$ and $\pi_{i j}$.

Therefore

and

$$
\begin{aligned}
& L_{1_{\rho}}=1, \quad L_{1_{\sigma}} \neq 1 \neq L_{1 \tau} ; \\
& L_{2_{\sigma}}=1, \quad L_{2_{\rho}} \neq 1 \neq L_{2_{\tau}} ; \\
& L_{3_{\tau}}=1, \quad L_{3_{\rho}} \neq 1 \neq L_{3_{\tau}} ; \\
& L_{12_{\rho}}=L_{12_{\sigma}}=1 \neq L_{12_{\tau}} ; \\
& L_{13_{\rho}}=L_{13_{\tau}}=1 \neq L_{13_{\sigma}} ;
\end{aligned}
$$

$$
L_{23_{\sigma}}=L_{23_{\tau}}=1 \neq L_{23_{\rho}} .
$$

Also, $\mathscr{L}_{1}=L_{1} L_{12} L_{13}, \mathscr{L}_{2}=L_{2} L_{12} L_{23}$ and $\mathscr{L}_{3}=L_{3} L_{13} L_{23}$.

In this new notation Lemma 3.12 (iii) asserts that $\left[\mathscr{L}_{1},\left(G_{\sigma:}\right) \hat{\pi}_{1}^{\prime}\right]_{1}^{\prime}=$ $\left[\mathscr{L}_{2},\left(G_{\rho_{\tau}}\right)_{\hat{\pi}_{2}^{\prime}}\right]=\left[\mathscr{L}_{3},\left(G_{\rho_{\sigma}}\right)_{\hat{\pi}_{3}^{\prime}}\right]=1$.

The unique $\alpha$-invariant Sylow 2-subgroup of $G$ will be denoted by $T$.

4. Soluble groups admitting a fixed-point-free automorphism. In Theorem 4.4, the main objective of this section, a type of structure theorem is established for a soluble group admitting a fixed-pointfree automorphism which involves star-subgroups of certain subgroups of the soluble group. Some consequences of this structure theorem will also be discussed.

Definition 4.1. $l$ is said to be a non-Fermat number if and only if

(i) $l$ is a positive integer; and

(ii) there does not exist an integer $m, m \geqq 1$, such that $2^{m}+1 \mid l$.

We remark that there exist infinitely many non-Fermat numbers which are square-free. 
Before stating the first lemma of this section, which contains the bulk of the work in proving Theorem 4.4, we recall that a group of automorphisms $A$ (of a group $G$ ) is said to act regularly on $G$ if all the nontrivial elements of $A$ act fixed-point-freely upon $G$. In Lemma 4.2 we shall use additive notation for modules.

Lemma 4.2. Suppose $G=P A$ where $P$ is a normal p-subgroup of $G$ ( $p$ a prime) and $A=\langle\alpha\rangle$ is cyclic with its order $a\{2, p\}^{\prime}-$ number. Let $V$ be an $\boldsymbol{F}_{q} G$-module which is faithful for $G\left(\boldsymbol{F}_{q}\right.$ denotes the finite field of $q$ elements, $q$ a prime) with $(q,|G|)=1$. Further, assume

(i) $P$ has a nontrivial $\alpha$-invariant section upon which $\langle\alpha\rangle$ acts regularly; and

(ii) if $p=2$, then either $|\langle\alpha\rangle|$ is a non-Fermat number or $P$ is abelian.

Then $C_{V}(\alpha) \neq 0$.

Proof. Assume the result is false and choose $G$ and $V$ to be a counter-example to the lemma, subject to $|G|+\operatorname{dim}_{F_{q}} V$ being minimal. Thus $C_{V}(\alpha)=0$. Note that a positive integer divisor of a non-Fermat number is also itself a non-Fermat number.

Suppose $F$ is a field which contains a copy of $\boldsymbol{F}_{q}$. Then it is well known and easy to show that

$$
\operatorname{dim}_{F_{q}}\left(C_{V}(\alpha)\right)=\operatorname{dim}_{F}\left(C_{\left(F \otimes F_{q} V\right)}(\alpha)\right)
$$

and so $C_{\left(F \otimes F_{q} V\right)}(\alpha)=0$. Thus, for the remainder of the proof of this lemma we shall assume $V$ to be a vector space over $F$ where $F$ is a field containing a copy of $\boldsymbol{F}_{q}$ and which is algebraically closed.

By hypothesis, there exists $\alpha$-invariant subgroups $Y$ and $Z$ of $P$ such that $Z \leqq Y$ and the induced action of $\langle\alpha\rangle$ upon $Y / Z$ is regular. Clearly $A Y$ and $V$ satisfy the hypothesis of the lemma and so, if $Y \neq P, C_{V}(\alpha) \neq 0$ since $|A Y|<|G|$, contrary to the choice of $G$ and $V$. Thus $Y=P$. Furthermore, it may be deduced that $Y / Z(=P / Z)$ has no nontrivial proper $\alpha$-invariant subgroups. For, suppose that $Y_{1}$ is a subgroup of $P$ containing $Z$ for which $Y_{1} / Z$ is a proper nontrivial $\alpha$-invariant subgroup of $Y / Z$, then, as the hypotheses of the lemma hold for $A Y_{1}$ and $V(\langle\alpha\rangle$ acts regularly on $\left.Y_{1} / Z\right)$, it may be asserted that $C_{V}(\alpha) \neq 0$. Thus there does not exist any such $Y_{1}$, as claimed. Hence $P / Z$ is an elementary abelian $p$-group and so, in particular, $\phi(P) \leqq Z$. In fact $\phi(P)=Z$. Suppose otherwise, and let bars denote images in $P / \phi(P)=\bar{P}$. Since $(|\langle\alpha\rangle|, p)=1$, appealing to Mashke's theorem (see, for example, [Theorem 3.3.1; 7]) gives that $\bar{P}=\bar{Z} \times \bar{Z}_{1}$ where $\bar{Z}_{1}$ is an $\alpha$-invariant subgroup of $\bar{P}$. Let $Z_{1}$ denote the inverse image of $\bar{Z}_{1}$ in $P$. Then $Z_{1}$ is a proper 
$\alpha$-invariant subgroup of $P$ which has $\langle\alpha\rangle$ acting regularly upon $Z_{1} / Z\left(=\bar{Z}_{1} \neq 1\right)$. Again, because of the minimal choice of $G$ and $V$, we obtain $C_{V}(\alpha) \neq 0$ which is untenable. Hence $\phi(P)=Z$, as asserted.

We next observe that the minimal choice of $G$ and $V$ forces $V$ to be an irreducible $F G$-module. If $V$ were not an irreducible $F G$ module, then use of Maschke's theorem gives $V=U_{1} \oplus U_{2} \oplus \cdots \oplus U_{d}$ where each $U_{i}$ is an irreducible $F G$-module and $d>1$. Since $G$ acts faithfully upon $V$ and $P$ is nontrivial (by assumption (i)), there exists at least one $U_{j}$ for which $P \not k \operatorname{ker} U_{j}$. In view of $P / \phi(P)$ being irreducible under the action of $\langle\alpha\rangle, P \cap \operatorname{ker} U_{j} \leqq \phi(P)$. Consequently

$$
\left[\left(\langle\alpha\rangle \cap \operatorname{ker} U_{j}\right), P\right] \leqq P \cap \operatorname{ker} U_{j} \leqq \dot{\phi}(P),
$$

and so the fact that $\langle\alpha\rangle$ is regular upon $P / \phi(P)$ demands that $\langle\alpha\rangle \cap$ $\operatorname{ker} U_{j}=1$. Therefore $\operatorname{ker} U_{j} \leqq P$ and so $\operatorname{ker} U_{j} \leqq \phi(P)$. Since $\operatorname{dim}_{F} U_{j}<\operatorname{dim}_{F} V$ and the pair $G / \operatorname{ker} U_{j}$ and $U_{j}$ satisfy the lemma's hypotheses, we may infer that $C_{U_{j}}(\alpha) \neq 0$, against the supposition $C_{V}(\alpha)=0$. Therefore $V$ is an irreducible $F G$-module.

Let $D$ be a nontrivial abelian normal $p$-subgroup of $G$. Then, an appeal to Clifford's theorem (see [Theorem 3.4.1; 7]) yields that $\left.V\right|_{D} \cong V_{1} \oplus \cdots \oplus V_{d}$ where each $V_{i}$ is itself the direct sum of irreducible $F D$-modules which are pairwise isomorphic (as $F D$-modules). The $V_{i}$ are normally referred to as the Wedderburn components of $V$ (with respect to $D$ ). For each $g \in G$, the mapping $m_{g}: V_{i} \mapsto V_{i} g=$ $\left\{v g \mid v \in V_{i}\right\}$ is a permutation upon the set of Wedderburn components of $V$ (with respect to $D$ ). Morerver, this permutation representation of $G$ (that is, $m: g \mapsto m_{g}$ ) upon the set of Wedderburn components of $V$ (with respect to $D$ ) is transitive.

The aim of the ensuing analysis is to show that the number of Wedderburn components of $V$ with respect to $D$ is one. So we shall suppose $d>1$, and argue for a contradiction. Since $d>1$, we may regard $V$ as being 'induced up' from a proper subgroup of $G$. More explicitly, if $\left\{1, g_{2}, \cdots, g_{d}\right\}$ is a set of right coset representatives of the stabilizer of $V_{1}$ in $G$ (in the permutation representation) on the Wedderburn components), then $V \cong V_{1} \otimes V_{1} g_{2} \otimes \cdots \otimes V_{1} g_{d}$ and so $V \cong V_{1}^{G}$.

Since the act of 'inducing up a module' is transitive (see [Theorem 4.4.4; 7]), we may suppose that $V \cong U^{G}$ where $U$ is an $H$-submodule of $V$ and $H$ is a maximal subgroup of $G$. We now consider two cases depending on whether $P \leqq H$ or $P \leqq H$.

Suppose that $H$ contains $P$. Then clearly $H=P(H \cap A)$ and $[G: H]$ is a prime number. Since $P \leqq G$, ker $U$ cannot contain $P$ (otherwise $P \leqq \operatorname{ker} V$; see [Theorem 4.4.3; 7]) and thus $P \cap \operatorname{ker} U \leqq P$. From 


\section{[ker $U \cap(H \cap A), P] \leqq \operatorname{ker} U \cap P \supsetneqq P$}

we may infer (using the regularity of $A$ upon $P / \dot{\phi}(P)$ and the fact that $\dot{\phi}(P)(\operatorname{ker} U \cap P)=P$ implies $P=\operatorname{ker} U \cap P)$ that $\operatorname{ker} U \cap(H \cap A)=1$. Thus ker $U \supsetneqq P$. Hence $C_{U}(H \cap A) \neq 0$ by applying induction to the pair $H /$ ker $U$ and $U$. Because $[G: H]$ is a prime it now follows (from the definition of the action of $G$ on the induced module) that $C_{r}(\alpha) \neq 0$ also.

Now we consider the case when $H$ does not contain $P$. Replacing $H$ by $H^{g},(g \in G)$ if necessary, and $U$ with $U^{g}$, the conjugate module, $\left(U^{i}\right.$ and $\left(U^{g}\right)^{G}$ will be isomorphic $F G$-modules) we may assume that $H=(P \cap H)(A \cap H)$. Then, if $A \supsetneqq H$, we have $H \supsetneqq P(A \cap H) \varsubsetneqq G$ which contradicts the maximality of $H$. Thus $A \leqq H$ and this, we claim, implies that $P \cap H=\dot{\phi}(P)$. For, by the irreducibility of $A$ on $P / \dot{\varphi}(P)$, either $(P \cap H) \dot{\varphi}(P)=P$ or $P \cap H \leqq \dot{\phi}(P)$. The former gives $P=P \cap H$ by a well-known property of $\dot{\phi}(P)$, and so $P \cap H \leqq \dot{\phi}(P)$ holds. Therefore

$$
H=(P \cap H)(H \cap A) \leqq \dot{\phi}(P)(H \cap A) \neq G,
$$

whence $P \cap H=\dot{\phi}(P)$ by the maximality of $H$. Let $g \in P \backslash \dot{\phi}(P)$ and set $g^{A}=\left\{g^{a}=a^{-1} g a \mid a \in A=\langle\alpha\rangle\right\}$. It is asserted that $g^{A}$ is contained in a set of right coset representatives for $\dot{\phi}(P)$ in $P$. Clearly, it will be sufficient to show that, if $g_{1}, g_{2} \in g^{4}$ with $g_{1}^{-1} g_{2} \in \phi(P)$, then $g_{1}=g_{2}$. So let $g_{1}, g_{2} \in g^{4}$ with $g_{1}^{-1} g_{2} \in \dot{\phi}(P)$. Since $g_{2}=g_{1}^{a}$ for some $a \in A, g_{1}^{-1} g_{1}^{a}=g_{1}^{-1} g_{2} \in \dot{\phi}(P)$. By choice, $g \notin \dot{\phi}(P)$ and so $g_{1} \notin \dot{\phi}(P)$. Thus $a=1$ by the regularity of $A$ upon $P / \phi(P)$. Hence $g_{2}=g_{1}^{a}=g_{1}$, as required. Now a complete set of right coset representatives for $\dot{\phi}(P)$ in $P$ is, at the same time (because $H \cap P=\dot{\phi}(P)$ ), a complete set of right coset representatives for $H$ in $G$. By choosing a complete set of right coset representatives for $\dot{\phi}(P)$ in $P$ containing $g^{4}$ for some $g \in P \backslash \dot{\phi}(P)$ and using the fact that $\alpha \in H$ and $\langle\alpha\rangle$ acts regularly upon $g^{1}$ it may be verified, using the definition of $U^{a}(=V)$, that $C_{V}(\alpha) \neq 0$ in this case also.

Hence the objective of showing that $d=1$ has been attained. Consequently, since $V_{\left.\right|_{D}}$ is a direct sum of isomorphic $F D$-modules and $F$ is algebraically closed, by [Lemma $3.2 .1 ; 7] D$ 'acts scalarly' upon $V$ and hence $G=C_{G}(D)$. A further inference from $d=1$, using [Theorem 3.2.3; 7], is that $D$ must be cyclic.

Therefore any characteristic abelian subgroup of $P$ must be cyclic, contained in $Z(P)$ and centralized by $A$. In particular, $P$ has class at most 2 (otherwise there would exist a characteristic abelian subgroup of $P$ which is not contained in $Z(P)$ ). From the regularity of $A$ on $P / \phi(P),([P, a] \dot{\phi}(P)) / \phi(P)=P / \dot{\phi}(P)$ for each $a \in A^{\sharp}$, and so $[P, a]=P$ for all $a \in A^{\sharp}$. This forces $C P(a) \leqq P^{\prime}$ for each $a \in A^{\sharp}$ for 
otherwise $C_{P}(a) \not P^{\prime}$ together with (2.3)(i) and (iii) applied to $P / P^{\prime}$ would yield $P \neq[P, a]$. Thus $A$ acts regularly upon $P / P^{\prime}$ and hence must act irreducibly upon $P / P^{\prime}$ (if not then the minimal choice of $G$ and $V$ would be contravened). So $P / P^{\prime}$ is an elementary abelian $p$-group and hence $\phi(P) \leqq P^{\prime} \leqq Z(P) \leqq C_{P}(A)(\neq P)$. The irreducibility of $A$ on $P / \phi(P)$ forces $\phi(P)=P^{\prime}=Z(P)=C_{P}(A)$. Let $x, y \in P$. Then (see [Lemma 2.2.2; 7]) $[x, y]^{p}=\left[x^{p}, y\right]=1$, since $x^{p} \in Z(P)$. Hence $P^{\prime}$ has exponent $p$ as well as being cyclic, and so $\left|P^{\prime}\right|=p$.

We are now in a position to use (2.15). So (in the notation of $(2.15))$

$$
\left.\chi\right|_{A}=\frac{\left(p^{m}-\delta\right) \rho}{|A|}+\delta \mu \quad(\delta=+1 \text { or }-1) .
$$

If $\delta=+1$, then $\left.\chi\right|_{A}$ would contain the regular character of $A$ as a constituent which then yields that $C_{V}(\alpha) \neq 0$. Thus $\delta=-1$. Moreover, $\left(p^{m}+1\right) /|A|>1$ would, again mean that $\left.\chi\right|_{A}$ has the regular character of $A$ as a constituent. Hence $p^{m}+1=|A|$. Now $|A|$ is odd by hypothesis and so the only possibility is $p=2$. Therefore $|A|$ is not a non-Fermat number and $P$ is not abelian, contrary to Hypothesis (ii).

This is the final contradiction, and so we conclude that $C_{V}(\alpha) \neq 0$.

REMARK. Results of a similar nature to Lemma 4.2 appear in [2] (Theorem 5.1(a)) and [18] (Theorem 4.1) and, in fact, our proof of Lemma 4.2 could be abbreviated by appealing to these two results.

To facilitate the statements of our next results we introduce

Hypothesis 4.3. Suppose $G$ is a soluble group admitting the automorphism $\alpha$ fixed-point-freely. Let $H$ and $K$ denote, respectively, the $\alpha$-invariant Hall $\pi$ - and $\pi^{\prime}$-subgroups of $G$ ( $\pi$ a set of primes), and suppose $\langle\beta\rangle$ is a subgroup of $\langle\alpha\rangle$ for which

(i) $C_{K}(\beta)=1$; and

(ii) $|\langle\beta\rangle|$ is odd and $(|\langle\beta\rangle|,|G|)=1$.

Further, if $2 \in \pi$, assume that either $H$ is abelian or $|\langle\beta\rangle|$ is a nonFermat number.

TheOrem 4.4. Assume Hypothesis 4.3 holds. Then $H / O_{\pi}(G)$ is star-covered with respect to $\langle\beta\rangle$.

Proof. Suppose the theorem is false, and let $G$ be a counterexample of minimal order. Since $(|G|,|\langle\beta\rangle|)=1$, by Lemma 3.3 (vi) and the minimal choice of $G, O_{\pi}(G)=1$. Thus, as the theorem is supposed false, $H$ is not star-covered with respect to $\langle\beta\rangle$ and so 
there exists an $\alpha$-invariant subgroup, $R$, of $H$ such that $R \neq R_{\langle\beta\rangle}^{*}$. Clearly we may assume that $R$ is a $p$-group for some $p \in \pi$.

By a result due to Hall and Higman ([Theorem 6.3.2; 7]) $C_{G}\left(O_{\pi^{\prime}},(G)\right) \leqq O_{\pi^{\prime}}(G)$. Thus $O_{\pi}\left(R O_{\pi^{\prime}}(G)\right)=1$. Hence $R O_{\pi^{\prime}}(G) \neq G$ would imply, because of the minimal choice of $G$, that $R$ is star-covered with respect to $\langle\beta\rangle$. Therefore, as $R \neq R_{\langle\beta\rangle}^{*}, R O_{\pi^{\prime}}(G)=G$. Suppose that $\left|\pi\left(O_{\pi^{\prime}}(G)\right)\right|>1$ and let $q \in \pi\left(O_{\pi^{\prime}}(G)\right)$. By $(2.1)(\mathrm{v})$, there exists $\alpha$ invariant Hall $\{p, q\}$ - and $q^{\prime}$-subgroups of $G$ which (respectively) take the form $R Q$ and $R Q_{1}$ where $Q$ and $Q_{1}$ are (respectively) the $\alpha$ invariant Hall $q$ - and $q^{\prime}$-subgroups of $O_{\pi^{\prime}}(G)$. Since $R Q$ and $R Q_{1}$ are proper subgroups of $G$ we have that both $R / C_{R}(Q)$ and $R / C_{R}\left(Q_{1}\right)$ are star-covered with respect to $\langle\beta\rangle$. Because $C_{G}\left(O_{\pi^{\prime}},(G)\right) \leqq O_{\pi^{\prime}}(G)$, $\mathrm{C}_{R}(Q) \cap C_{R}\left(Q_{1}\right)=1$. Consequently $C_{R}(Q) \cong\left(C_{R}(Q) C_{R}\left(Q_{1}\right)\right) / C_{R}\left(Q_{1}\right) \quad$ (and this isomorphism commutes with $\alpha)$, and so $C_{R}(Q)$ is star-covered with respect to $\langle\beta\rangle$. Applying Lemma 3.3 (vii) we conclude that $R$ is star-covered with respect to $\langle\beta\rangle$, contrary to $R \neq R_{\langle\beta\rangle}^{*}$. Hence we deduce that $\left|\pi\left(O_{\pi^{\prime}}(G)\right)\right|=1$, and so $G=R O_{q}(G)$ (where $q$ is a prime number).

Observe that, as $R \neq R_{\langle\beta\rangle}^{*}$ and $(|G|,|\langle\beta\rangle|)=1$, there exists a nontrivial $\alpha$-invariant section of $R$ upon which $\langle\beta\rangle$ acts regularly, namely $R / \phi(R) R_{\langle\beta\rangle}^{*}$. Note that, by [Theorem $\left.5.1 .4 ; 7\right],\langle\beta\rangle R$ acts faithfully upon $O_{q}(G) / \phi\left(O_{q}(G)\right)$. An examination of $G$ yields that the necessary hypotheses exist for an application of Lemma 4.2 with $A=\langle\beta\rangle, P=R$ and $V=O_{q}(G) / \phi\left(O_{q}(G)\right)$. Consequently $C_{V}(\beta) \neq 1$. Hence, as $(q,|\langle\beta\rangle|)=1, C_{0_{q}(G)}(\beta) \neq 1$ by $(2.3)\left(\right.$ iii). However, $C_{K}(\beta)=1$ by hypothesis and so we have obtained a contradiction. Thus there does not exist a counterexample to the theorem, so establishing the theorem.

REMARKS. (i) Theorem 4.4 may be viewed as an extension of results such as $(2.13)$ and [Theorem $3.3 ; 14$ ]. It may also be considered as a (weak) analogue of (2.3)(iv).

(ii) In Theorem 4.4 the presence of the fixed-point-free automorphism is not strictly necessary.

(iii) The following example shows that the 'non-Fermat number' assumption in Theorem 4.4 is necessary.

Let $R$ denote a 2 -group of order $2^{6}$ with the following properties. (For the existence and properties of such a group see [8]-actually $R$ is isomorphic to a Sylow 2-subgroup of $U_{3}(4)$.)

(i) $Z(R)=R^{\prime}=\phi(R)$ is a Klein four group;

(ii) $R$ possesses an automorphism $\alpha$ of order 15 (set $\rho=\alpha^{5}$ and $\left.\sigma=\alpha^{3}\right)$

(iii) $\rho$ acts fixed-point-freely upon $R$; and

(vi) $C_{R}(\sigma)=Z(R)$. 
Let $x \in Z(R)$. Then $\bar{R}=R /\langle x\rangle$ is an extra-special group which admits $\sigma$ with $C_{\bar{R}}(\sigma)=Z(\bar{R})$. We claim that there exists an $\boldsymbol{F}_{q} \bar{R}$ module $U$ faithful for $\bar{R}, q \notin\{2,3,5\}$, and such that $U$ extends to an $\boldsymbol{F}_{q}(\bar{R}\langle\sigma\rangle)$ module with $\sigma$ acting fixed-point-freely upon $U$. Let $q \notin$ $\{2,3,5\}$ be a prime number such that $\boldsymbol{F}_{q}$ is a splitting field for $\langle\sigma\rangle \bar{R}$ and $\boldsymbol{F}_{q}$ contains a 5 th root of unity. Let $W$ be an irreducible $\boldsymbol{F}_{q}(\bar{R}\langle\boldsymbol{\sigma}\rangle)$ module faithful for $\bar{R}\langle\boldsymbol{\sigma}\rangle$. Since $\bar{R}\langle\boldsymbol{\sigma}\rangle$ has a unique minimal normal subgroup, there exists such a $W$. Let $F$ be the algebraic closure of $\boldsymbol{F}_{q}$, and set $W_{F}=W \otimes_{F_{q}} F$. Then $W_{F}$ is an irreducible $F(\bar{R}\langle\sigma\rangle)$ module faithful for $\bar{R}\langle\sigma\rangle$. Let $\chi$ denote the character of $W_{F}$. By (2.15), since $\left(p^{m}-\delta\right) /|A|=(4-\delta) / 5$ must be an integer, $\chi_{\left.\right|_{\langle\sigma\rangle}}=\rho-\mu$ where $\rho$ is the regular character of $\langle\sigma\rangle$ and $\mu$ is some irreducible character of $\langle\sigma\rangle$. Let $\hat{\mu}$ denote the inverse of $\mu$ in the character group of $\langle\sigma\rangle$. We may regard $\hat{\mu}$ as a character of $\bar{R}\langle\sigma\rangle$ (note that $\hat{\mu}$ takes values in $\boldsymbol{F}_{q}$ ). Let $\hat{M}$ be an $\boldsymbol{F}_{q}(\bar{R}\langle\boldsymbol{\sigma}\rangle$ ) module affording $\hat{\mu}$. If $\mu$ is the trivial character, then take $U=W$. Otherwise take $U=W \otimes_{F_{q}} \hat{M}$. Then $U$ has the required properties.

So $U$ may be considered as a module for $R\langle\sigma\rangle$ with ker $U=\langle x\rangle$. Set $V=U^{R\langle\alpha\rangle}$. Since ker $U \nRightarrow R\langle\alpha\rangle, R\langle\alpha\rangle$ will be represented faithfully on $V$. Hence, setting $G=R V$, we have $O_{2}(G)=1$. It may be checked that $C_{V}(\alpha)=1$ and so $G$ admits $\alpha$ fixed-point-freely. Further $R^{*}=C_{R}(\sigma)=Z(R) \neq R$, and so $G$ is an example of the required type.

Corollary 4.5. Assume Hypothesis 4.3 holds and let $R$ be an $\alpha$-invariant subgroup of $H$. Then $R=R_{\langle\beta\rangle}^{*}\left(O_{\pi}(G) \cap R\right.$ ) (and, in particular, $H=H_{\langle\beta\rangle}^{*} O_{\pi}(G)$ ).

Proof. This follows from Theorem 4.4 and Lemma 3.3 (vi).

Typically, in the situations to which Corollary 4.5 will be applied $H$ will be an $\alpha$-invariant nilpotent Hall subgroup and so, sometimes, the following lemma will be of use.

Lemma 4.6. Assume Hypothesis 4.3 holds. Moreover, suppose $H$ is nilpotent and $R$ is an $\alpha$-invariant subgroup of $H$ which contains $O_{\pi}(G)$. If $\left(N_{H}(R)\right)_{\langle\beta\rangle}^{*} \leqq R$, then $R=H$.

Proof. From Corollary 4.5,

$$
\begin{aligned}
N_{H}(R) & =\left(N_{H}(R)\right)_{\langle\beta\rangle}^{*}\left(N_{H}(R) \cap O_{\pi}(G)\right) \\
& =\left(N_{H}(R)\right)_{\langle\beta\rangle}^{*} O_{\pi}(G) \\
& \leqq R,
\end{aligned}
$$


and so $R=N_{H}(R)$. The fact that $H$ is nilpotent forces $R=H$, as desired.

The last result of this section gives a hint of the type of role the 'star-subgroup' will play in global arguments.

Lemma 4.7. Let $(G, A)$ be a pair satisfying Hypothesis I with $A=\langle\alpha\rangle$ cyclic, $|A|$ odd and $(|G|,|A|)=1$. Let $P$ denote the $\alpha$ invariant Sylow p-subgroup of $G$, and assume that either $p \neq 2$ or $|A|$ is a non-Fermat number. Suppose $H$ and $K$ are two proper $\alpha$ invariant subgroups of $G$ which contain $P$. If $P$ is not star-covered, then $O_{p}(H) \cap O_{p}(K) \neq 1$.

Proof. Since $H$ and $K$ are $\alpha$-invariant soluble groups, Corollary 4.5 applies to both $H$ and $K$, and so $P / O_{p}(H)$ and $P / O_{p}(K)$ are both star-covered. If $O_{p}(H) \cap O_{p}(K)=1$, then $O_{p}(H) \cong O_{p}(H) O_{p}(K) / O_{p}(K)$ whence $O_{p}(H)$ is star-covered. By Lemma 3.3 (vii) this implies $P$ is star-covered, contrary to the lemma's hypotheses. Therefore $O_{p}(H) \cap$ $O_{p}(K) \neq 1$.

5. Elementary properties of pairs satisfying Hypothesis I. Throughout this section, $(G, A)$ will assumed to be a pair satisfying Hypothesis I. For the duration of this section $L, M$ and $N$ will denote (respectively) $A$-invariant nilpotent Hall $\lambda$-, $\mu$ - and $\eta$-subgroups of $G$ with $\lambda, \mu$ and $\eta$ pairwise disjoint. Set $\mathscr{P}_{L}(M)=X$ and $\mathscr{P}_{M}(L)=Y$. Our first result contains some straightforward observations, which will be of frequent use.

Lemma 5.1. Suppose $L M \neq M L$ and let $\beta \in A^{\#}$ with $|\langle\beta\rangle|$ a prime number and $|\langle\beta\rangle| \notin \lambda \cup \mu$.

(a) If $M_{\beta} \leqq Y$, then $M=Y C_{M}([J, \beta])$ for all $A$-invariant abelian subgroups $J$ of $X$. Moreover, if $2 \notin \lambda$, then $M=Y C_{M}([X, \beta])$.

(b) If $M_{\beta} \leqq Y$, then $Z(L) \cap X \leqq L_{\beta}$.

(c) If $M_{\beta} \leqq Y, 2 \notin \lambda,[X, \beta]=X$ and $|\mathscr{L C}(\lambda, \mu)|=2$ then $X=1$.

For (d) and (e) assume, additionally, that $A$ is cyclic of squarefree order. Suppose $\gamma \in A^{\sharp}$.

(d) If $M_{\langle r\rangle}^{*} \leqq Y,\langle\gamma\rangle$ is a $\mu^{\prime}$-group and $J$ is an A-invariant subgroup of $X$ for which $J_{r}=1$, then $M=Y C_{m}(J)$.

(e) If $M_{\langle\gamma\rangle}^{*} \leqq Y$ and $\langle\gamma\rangle$ is a $(\lambda \cup \mu)^{\prime}$-group, then $Z(L) \cap X \leqq Z(L)_{\langle\gamma\rangle}^{*}$.

Proof. (a) Applying (2.14)(i) to $O_{\mu}(M X) J$ yields, since $J$ normalizes $O_{\mu}(M X) \cap Y\left(\geqq O_{\mu}(M X)_{\beta}\right)$, that

$$
O_{\mu}(M X)=C_{o_{\mu}(M X)}([J, \beta])\left(O_{\mu}(M X) \cap Y\right) .
$$

Now (2.20) gives $M=Y C_{M}([J, \beta])$. Similarly, it may be verified 
that $M=Y C_{M}([X, \beta])$ when $2 \notin \lambda$.

(b) Employing (a) with $J=Z(L) \cap X$ gives that $M=Y C_{M}([Z(L) \cap$ $X, \beta])$. Since $Y \neq M$ by hypothesis, $Z(L) \cap X \leqq L_{\beta}$ must hold.

(c) From (a), $M=Y C_{M}([X, \beta])=Y C_{M}(X)$ whence, by (2.21)(vi), $X=1$.

(d) Using (2.14)(ii) on $O_{\mu}(M X) J$ and then employing (2.20) yields (d).

(e) Suppose $|\langle\gamma\rangle|=r_{1}, \cdots, r_{m}$ (where the $r_{i}$ are prime numbers). Then (using the notation of $\S 3)(Z(L) \cap X)_{\langle\gamma\rangle}^{*}=\left\langle(Z(L) \cap X)_{\alpha_{i}}\right| i=$ $1, \cdots, m\rangle$ by Lemma $3.3(\mathrm{v})$. Set $Z=\left[\cdots,\left[\left[Z(L) \cap X, \alpha_{1}\right], \cdots\right], \alpha_{m}\right]$. From (2.3)(i) $Z(L) \cap X=Z \times(Z(L) \cap X)_{\langle\gamma\rangle}^{*}$. Clearly $Z_{\langle\gamma\rangle}^{*}=1$ and, in view of (d), we must have $Z=1$ since $L M \neq M L$. This proves (e).

Lemma 5.2. Suppose $L$ and $M$ do not permute. Also, suppose that $B$ is a $(\lambda \cup \mu)^{\prime}$-group of $A$ and that $O_{\mu}(L Y) \neq 1$. Then either

(a) $\left[O_{\mu}(M X) \cap Y, B\right] \neq 1$; or

(b) $M X \leqq C_{G}(B)$.

Proof. Since $\quad O_{\mu}(L Y)\left(O_{\lambda}(L Y) \cap X\right) \leqq L Y \cap M X, \quad O_{\mu}(L Y)=1$, $O_{\lambda}(L Y) \neq 1$ (by $(2.20)$ ) and $L Y \neq M X$, we see, because of (2.18), that $O_{\lambda}(L Y) \cap X=1$. By (2.21)(ii) $F(X Y)=O_{\mu}(M X) \cap Y=O_{\mu}(X Y)$. Thus $C_{X Y}\left(O_{\mu}(M X) \cap Y\right) \leqq O_{\mu}(M X) \cap Y$ since $X Y$ is soluble (by (2.5)).

Now suppose that $\left[O_{\mu}(M X) \cap Y, B\right]=1$ holds. Then, using $(2.3)(\mathrm{x})$ on $X Y$, yields that

$$
[X Y, B] \leqq C_{X Y}\left(O_{\mu}(M X) \cap Y\right) \leqq O_{\mu}(M X) \cap Y \leqq C_{M}(B) .
$$

Thus $[X Y, B]=[[X Y, B], B]=1 . \quad$ By $\quad(2.21)($ iv $) \quad C_{M}(Y) \leqq Y$ whence, since $Y \leqq C_{M}(B), M \leqq C_{M}(B)$ by $(2.3)(\mathrm{v})$.

Therefore, either $\left[O_{\mu}(M X) \cap Y, B\right] \neq 1$ or $M X \leqq C_{G}(B)$ must hold.

Lemma 5.3. Suppose that $L$ and $M$ do not permute. Further, assume that $A=\langle\alpha\rangle$ is a cyclic $(\lambda \cup \mu)^{\prime}$-group of square-free order. If $O_{\mu}(L Y) \neq 1$ and $2 \notin \mu$, then $X=1$.

Proof. We recall (see $\S 3$ ) that $\alpha_{i}$ denotes a generator of the Sylow $r_{i}$-subgroup of $\langle\alpha\rangle$ for each $i \in \Lambda=\{1, \cdots, n\}$ where $|\langle\alpha\rangle|=$ $r_{1}, \cdots, r_{n}$ (with each $r_{i}$ a prime).

By (2.22), $\mathscr{L}(\lambda, \mu)=\{L Y, M X\}$. Thus, since $G_{\alpha_{i}}$ is $\alpha$-invariant by $(2.3)(\mathrm{vi}),\left(G_{\alpha_{i}}\right)_{\{\lambda \cup \mu\}}$ is contained in at least one of $L Y$ and $M X$ (note that $G \neq G_{\alpha_{i}}$ because of Hypothesis I (iv)). That is, for each $i \in \Lambda$, either $L_{\alpha_{i}} \leqq X$ or $M_{\alpha_{i}} \leqq Y$. Set $\Delta=\left\{i \in \Lambda \mid L_{\alpha_{i}} \leqq X\right\}$ and set $\Gamma=\left\{i \in \Delta \mid\left[O_{\mu}(M X) \cap Y, \alpha_{i}\right]=1\right\}$. Also, let $\gamma=\prod_{i \in \Gamma} \alpha_{i}$ and let $\beta=\prod_{i \notin \Gamma} \alpha_{i}$. 
From Lemma 5.2, $M X \leqq G_{\alpha_{i}}$ for each $i \in \Gamma$, and so $M X=\left(G_{\gamma}\right)_{\{\lambda \cup \mu\}}$. Hence $\beta$ acts fixed-poin-freely upon $M X$. Let $i \in \triangle \backslash \Gamma$, then, since $2 \notin \mu, L=X C_{L}\left(\left[O_{\mu}(M X) \cap Y, \alpha_{i}\right]\right)$ by Lemma $5.1(\mathrm{a})$.

Because $i \in \triangle \backslash \Gamma,\left[O_{\mu}(M X) \cap Y, \alpha_{i}\right] \neq 1$ whence, since $|\mathscr{L}(\lambda, \mu)|=2$,

$$
N_{M}\left(\left[O_{\mu}(M X) \cap Y, \alpha_{i}\right]\right) \leqq Y .
$$

Thus, employing $(2.3)\left(\right.$ viii) (and setting $N=N_{o_{\mu}(M X)}\left(O_{\mu}(M X) \cap Y\right)$ ) yields that

$$
\begin{aligned}
N_{\alpha_{i}} & \leqq N_{M}\left(\left[O_{\mu}(M X) \cap Y, \alpha_{i}\right]\right) \cap O_{\mu}(M X) \\
& \leqq Y \cap O_{\mu}(M X) \quad(\text { for each } i \in \Delta \backslash \Gamma) .
\end{aligned}
$$

Now $M_{\alpha_{i}} \leqq Y$ for all $i \notin \Delta$ and so $N_{\langle\beta\rangle}^{*} \leqq Y \cap O_{\mu}(M X)$ by Lemma $3.3(\mathrm{v})$. Thus $\left(N / Y \cap O_{\mu}(M X)\right)_{\langle\beta\rangle}^{*}=1$ by Lemma $3.3(\mathrm{vi})$. Since $X$ normalizes both $N$ and $Y \cap O_{\mu}(M X)$, by applying (2.8) to the semidirect product $X\left(N / Y \cap O_{\mu}(M X)\right.$ ) (recall that $\beta$ acts fixed-point-free upon $M X)$ we obtain

$$
N=C_{N}(X)\left(Y \cap O_{\mu}(M X)\right) .
$$

Now suppose that $X \neq 1$. Then $C_{N}(X) \leqq Y$ since $|\mathscr{L}(\lambda, \mu)|=2$. Hence $N_{o_{\mu}(M X)}\left(O_{\mu}(M X) \cap Y\right)=N=O_{\mu}(M X) \cap Y$ which then yields that $O_{\mu}(M X) \leqq Y$. In view of (2.20) and the assumption $L M \neq M L$ this is untenable.

Therefore we conclude that $X=1$, so establishing the lemma.

REMARKs. The proof of Lemma 5.3 is capable of several variations. For example, by using Lemma 2.9 of [13] instead of (2.8) in the proof of Lemma 5.3, we obtain the following which extends [Corollary $3.13 ; 13$ ].

Lemma 5.4. Suppose that $L M \neq M L$ and that $A$ is an abelian $(\lambda \cup \mu)^{\prime}$-group of square-free exponent. If $O_{\mu}(L Y) \neq 1$ and $2 \notin \mu$, then $X=1$.

For Lemmas 5.5 and 5.7 and Corollary 5.6 we assume that $A$ is an abelian $(\lambda \cup \mu)^{\prime}$-group of square-free exponent.

LEMMA 5.5. If $L M \neq M L, 2 \notin \mu$ and $L_{\beta} \leqq X$ where $\beta \in A^{\sharp}$ with $|\langle\beta\rangle|$ a prime number, then either

(i) $Y \leqq M_{\beta}$; or

(ii) $L_{\beta}=X=1$.

Proof. By combining Lemma 5.4 and (2.23). 
We single out a special instance of Lemma 5.5.

Corollary 5.6. Suppose $L M \neq M L$ and let $\beta \in A^{\sharp}$ be such that $|\langle\beta\rangle|$ is a prime number. If $L_{\beta}=1,2 \notin \mu$ and $X \neq 1$, then $Y \leqq M_{\beta}$.

Lemma 5.7. Suppose that $L M \neq M L$. If $L_{\beta}=1$ where $\beta \in A^{\#}$ with $|\langle\beta\rangle|$ a prime number and $2 \notin \mu$, then $Y=N_{M}(L)$.

Proof. Clearly $N_{M}(L) \leqq Y$. We proceed to show the reverse inequality. From (2.13)(ii) $L Y$ has $\lambda$-length at most one. Thus $Y=O_{\mu}(L Y) N_{M}(L)$.

If $O_{\mu}(L Y)=1$, then the required conclusion follows. While $O_{\mu}(L Y) \neq 1$ implies that $X=1$ by Lemma 5.5 which in turn gives, by (2.20), $L=O_{\lambda}(L Y) X=O_{\lambda}(L Y)$. Hence, in this case also, we have $Y \leqq N_{M}(L)$. Now the lemma follows.

The remaining three results of this section are the so called 'triangle lemmas'. They play a fundamental role in connecting the local and global situations that we shall encounter.

LEMMA 5.8. ('First Triangle lemma'). Let $\beta \in A^{\sharp}$ with $|\langle\beta\rangle| a$ prime number and $|\langle\beta\rangle| \notin \lambda \cup \mu \cup \eta$. Suppose that following hold:

(i) $L$ permutes with both $M$ and $N$;

(ii) $M N \neq N M$; and

(iii) $N(M L) \neq G$.

Then the following statements hold.

(a) If $M_{\beta} \leqq \mathscr{P}_{M}(N)$, then

$$
O_{\mu}(M L)=C_{o_{\mu}(M L)}([J, \beta])\left(O_{\mu}(M L) \cap \mathscr{P}_{M}(N)\right)
$$

for all A-invariant abelian subgroups $J$ of $L$.

(b) If $M_{\beta} \leqq \mathscr{P}_{M}(N)$ and $2 \notin \lambda$, then

$$
O_{\mu}(M L)=C_{o_{\mu}(M L)}([L, \beta])\left(O_{\mu}(M L) \cap \mathscr{P}_{M}(N)\right) .
$$

(c) Suppose $\beta \in Z(A)$ and $|\mathscr{M}(\mu, \eta)|=2$. If $M_{\beta} \leqq \mathscr{P}_{M}(N)$, then either $J \leqq L_{\beta}$ for all characteristic abelian subgroups $J$ of $O_{\lambda}(L N)$ or at least one of

$$
O_{\mu}(M L) \leqq \mathscr{P}_{M}(N) \quad \text { and } \quad N_{\beta} \leqq \mathscr{P}_{N}(M)
$$

must hold.

Further, if $2 \notin \lambda$, then either $O_{\lambda}(L N) \leqq L_{\beta}$ or at least one of

$$
O_{\mu}(M L) \leqq \mathscr{P}_{M}(N) \quad \text { and } \quad N_{\beta} \leqq \mathscr{P}_{N}(M)
$$

must hold. 
For (d) and (e) we assume, additionally, that $A=\langle\alpha\rangle$ is cyclic of square-free order. Let $\langle\gamma\rangle$ be a ( $\lambda \cup \mu)^{\prime}$-subgroup of $A$.

(d) If $M_{\langle\gamma\rangle}^{*} \leqq \mathscr{P}_{M}(N)$, then, for all $\alpha$-invariant subgroups $J$ of $L$ for which $J_{\gamma}=1$,

$$
O_{\mu}(M L)=C_{o_{\mu}(M L)}(J)\left(O_{\mu}(M L) \cap \mathscr{P}_{M}(N)\right) .
$$

(e) Suppose that $M_{\langle\gamma\rangle}^{*} \leqq \mathscr{P}_{M}(N)$ and that $L_{\gamma}=1$. If $2 \notin \mu$, then

(i) $\quad M=\mathscr{P}_{M}(N) C_{M}(L)$; and

(ii) $N_{N}(J) \leqq \mathscr{P}_{N}(M)$ for all nontrivial $\alpha$-invariant subgroups $J$ of $L$.

(f) Suppose that $M_{\langle\gamma\rangle}^{*} \leqq \mathscr{P}_{M}(N)$ and that $L_{\gamma}=1$. Then either $O_{\lambda}(L N)=1$ or $2 \in \mu$.

Proof. Firstly, we observe that $\mathscr{P}_{M L}(N) N \neq G$ since $\mathscr{P}_{M L}(N) N=G$ forces (because of order considerations) $G=(M L) N$, contrary to assumption (iii). $\quad$ By $(2.26), \mathscr{P}_{M L}(N)=\mathscr{P}_{M}(N) \mathscr{P}_{L}(N)=\mathscr{P}_{M}(N) L$. Consequently $L$ normalizes both $O_{\mu}(M L)$ and $O_{\mu}(M L) \cap \mathscr{P}_{M}(N)$. Now (a) and (b) follow by applying (2.14)(i), respectively, to $O_{\mu}(M L) J$ and $O_{\mu}(M L) L$. By using (2.14)(ii) upon $O_{\mu}(M L) J$ we also obtain (d).

We now proceed to establish (c). Let $J$ be a characteristic abelian subgroup of $O_{\lambda}(L N)$ with $[J, \beta] \neq 1$. Employing part (a) gives

$$
O_{\mu}(M L)=C_{o^{\prime}(M L)}([J, \beta])\left(O_{\mu}(M L) \cap \mathscr{P}_{M}(N)\right) .
$$

Since $N_{\beta}$ normalizes $J$, we have $N_{\beta} \leqq N_{L N}([J, \beta])$ by (2.3)(viii). Thus considering $\left(N_{G}([J, \beta])\right)_{\{\mu \cup \eta\}}$ in conjunction with the hypothesis that $|\mathscr{M}(\mu, \eta)|=2$, we infer that either $C_{M}([J, \beta]) \leqq \mathscr{F}_{M}(N)$ or that $N_{\beta} \leqq \mathscr{P}_{N}(M)$. Hence the first part of (c) follows; the second part is established similarly (using (b) in place of (a)).

(e) By part (d) we have

$$
O_{\mu}(M L)=C_{o_{\mu}(M L)}(L)\left(O_{\mu}(M L) \cap \mathscr{P}_{M}(N)\right) .
$$

Since, by hypothesis, $2 \notin \mu$ employing Corollary 4.5 (note $L_{\beta}=1$ ) gives

$$
M=M_{\langle\beta\rangle}^{*} O_{\mu}(M L) .
$$

Thus

$$
M \leqq \mathscr{P}_{M}(N) C_{M}(L) \leqq M
$$

which gives (e)(i).

If $N_{N}(J) \not \mathscr{P}_{N}(M)$ for some nontrivial $\alpha$-invariant subgroup $J$ of $L$, then, since $\mathscr{C}(\mu, \eta)=\left\{M \mathscr{P}_{N}(M), N \mathscr{P}_{M}(N)\right\}$ by $(2.22), N_{M}(J) \leqq$ $\mathscr{P}_{M}(N)$. In particular, $C_{M}(L) \leqq \mathscr{P}_{M}(N)$ so forcing the untenable $M=\mathscr{P}_{M}(N)$. Thus (e)(ii) holds. 
(f) Suppose that $O_{\lambda}(L N) \neq 1$. If, further, $2 \notin \mu$, then (e)(ii) would demand (with $J=O_{\lambda}(L N)$ ) that $N \leqq \mathscr{P}_{N}(M)$, a contradiction. Hence (f) follows.

Lemma 5.9. ('Second Triangle Lemma'). Let $\langle\beta\rangle$ be a cyclic $(\lambda \cup \mu)^{\prime}$-subgroup of $A$ which is of square-free order. Assume that

(i) $M N \neq N M$ and $L M \neq M L$;

(ii) $M_{\langle\beta\rangle}^{*} \leqq \mathscr{P}_{M}(N)$; and

(iii) $J$ is a nontrivial A-invariant subgroup of $\mathscr{P}_{Z}(N)$ such that $J_{\beta}=1$ (here we have set $Z=\mathscr{P}_{L}(M)$ ).

Then

(a) $\quad M=C_{M}(J) \mathscr{P}_{M}(N) \mathscr{P}_{M}(L)$; and

(b) if $M \leqq M Z$, then $M=C_{M}(J) \mathscr{P}_{M}(N)$.

Proof. (a) If it were the case that $N \mathscr{P}_{M Z}^{\circ}(N)=G$, then order considerations would force $L=Z=\mathscr{P}_{L}(M)$ contrary to the supposition $M L \neq L M$. Hence, using (2.26), we have that $\mathscr{P}_{M Z}(N)=$ $\mathscr{P}_{M}(N) \mathscr{P}_{Z}(N)$. Therefore $J$ normalizes both $O_{\mu}(M Z)$ and $O_{\mu}(M Z) \cap$ $\mathscr{P}_{M}(N)\left(\geqq O_{\mu}(M Z)_{\langle\beta\rangle}^{*}\right)$ whence, by $(2.14)(\mathrm{ii})$,

$$
O_{\mu}(M Z)=C_{o_{\mu}(M Z)}(J)\left(O_{\mu}(M Z) \cap \mathscr{P}_{M}(N)\right) .
$$

Thus, using (2.20),

$$
M=\mathscr{P}_{M}(L) O_{\mu}(M Z)=C_{M}(J) \mathscr{P}_{M}(L) \mathscr{P}_{M}(N),
$$

as required.

(b) Arguing as in part (a) we obtain

$$
M=O_{\mu}(M Z)=C_{M}(J)\left(M \cap \mathscr{P}_{M}(N)\right)=C_{M}(J) \mathscr{P}_{M}(N),
$$

and we have (b).

LEMMA 5.10. ('Third Triangle Lemma'). Let $\langle\beta\rangle$ be a cyclic $(\lambda \cup \mu)^{\prime}$-subgroup of $A$ which is of square-free order. Also assume

(i) $N M \neq M N$ and $L M \neq M L$ (set $Z=\mathscr{P}_{L}(M)$ ); and

(ii) $\quad M_{\langle\beta\rangle}^{*} \leqq \mathscr{P}_{M}(N)$.

Then the following statements hold.

(a) If $J$ is a nontrivial A-invariant subgroup of $\mathscr{P}_{z}(N)$ with $J_{\beta}=1$ and one of $\mathscr{P}_{M}(N) \leqq \mathscr{P}_{M}(L)$ and $\mathscr{P}_{M}(L) \leqq \mathscr{P}_{M}(N)$ holds, then $C_{M}(J) \$ \mathscr{P}_{M}(L)$ and $C_{M}(J) \not \mathscr{P}_{M}(N)$.

(b) If $A$ is abelian of square-free exponent, $L_{\beta}=1, J$ is a nontrivial A-invariant subgroup of $\mathscr{P}_{Z}(N)$ and $2 \notin \mu$, then $C_{M}(J) \not$ $\mathscr{F}_{M}(L)$.

(c) If $Z(L)_{\beta}=1$ and one of $\mathscr{P}_{M}(N) \leqq \mathscr{P}_{M}(L)$ and $\mathscr{P}_{M}(L) \leqq \mathscr{P}_{M}(N)$ holds, then $\mathscr{P}_{Z}(M) \cap Z(L)=1$. 
(d) If $A$ is abelian of square-free exponent, $L_{\beta}=1$ and $2 \notin \mu$, then $\mathscr{P}_{Z}(M) \cap Z(L)=1$.

Proof. (a) By Lemma $5.9(\mathrm{a}), \quad M=C_{M}(J) \mathscr{P}_{M}(N) \mathscr{P}_{M}(L)$. Since, by hypothesis, one of $\mathscr{P}_{M}(L) \geqq \mathscr{P}_{M}(N)$ and $\mathscr{P}_{M}(N) \geqq \mathscr{P}_{M}(L)$ must hold and $M$ does not permute with either $L$ or $M$, it follows that $C_{M}(J) \not \mathscr{P}_{M}(L)$ and $C_{M}(J) \not \mathscr{P}_{M}(N)$.

(b) In view of part (a), we may suppose that $\mathscr{P}_{M}(L) \not \mathscr{P}_{M}(N)$. Since $M_{\langle\beta\rangle}^{*} \leqq \mathscr{P}_{M}(N)$, this gives $\mathscr{P}_{M}(L) \neq \mathscr{P}_{M}(L)_{\langle\beta\rangle}^{*}$ and so, since $2 \notin \mu$, Corollary 4.5 dictates that $O_{\mu}\left(\mathscr{P}_{M}(L) L\right) \neq 1$. From Lemma 5.4 (since $2 \notin \mu$ and $L M \neq M L), Z=\mathscr{P}_{L}(M)=1$. However, by hypothesis $1 \neq J \leqq \mathscr{P}_{Z}(N) \leqq Z$. This establishes (b).

(c) If $Z(L) \cap \mathscr{P}_{Z}(M)$ were nontrivial, then setting $J=Z(L) \cap$ $\mathscr{P}_{Z}(M)$ would violate the conclusion of (a). Thus $Z(L) \cap \mathscr{P}_{Z}(M)=1$.

(d) Likewise, (d) follows from the assertion contained in (b).

6. Normal p-complements. This short section consists of criteria for the existence of normal $p$-complements in groups which admit certain automorphism groups.

Lemma 6.1. Let $G$ be a finite group admitting a coprime automorphism group $A$. If $C_{G}(A)$ contains a Sylow p-subgroup of $G$ and $C_{G}(A)=O_{p^{\prime}, p}\left(C_{G}(A)\right)$, then $G=O_{p^{\prime}, p}(G)$.

Proof. Let $R$ be a nontrivial subgroup of $P$ where $P \in \operatorname{Syl}_{p} G$ and $P \leqq C_{G}(A)=C$. From (2.3)(xii) $N_{G}(R)=N_{G}(R) C_{G}(R)$ and so $N_{G}(R) / C_{G}(R) \cong N_{C}(R) / C_{C}(R)$. Because $C$ has a normal $p$-complement, $N_{C}(R) / C_{C}(R)$ must be a p-group. Now a well-known results of Frobenius ([Theorem 7.4.5; 7]) yields the desired conclusion.

Our next result will render valuable service in Part IV.

LeMma 6.2. Suppose $G$ is a finite group admitting the coprime automorphism $\gamma$, which is of square-free order $r_{1}, \cdots, r_{m}\left(r_{i}\right.$ a prime for $i=1, \cdots, m)$. Further, assume that the following hold:

(i) $G=T H$ where $T$ is a $\gamma$-invariant Sylow 2-subgroup of $G$ and $H$ is a $\gamma$-invariant Hall 2 '-subgroup of $G$;

(ii) $C_{G}(\gamma)=C_{T}(\gamma)$;

(iii) $C_{T}(\beta) \leqq N_{T}(H)$ for all $\beta \in\langle\gamma\rangle^{\#}$;

(iv) $N_{G}(J(T))$ and $C_{G}(Z(T))$ both possess normal 2-complements; and

( $\mathrm{v})$ every finite group admitting a coprime fixed-point-free automorphism of square-free order $s_{1}, \cdots, s_{m}$ ( $s_{i}$ a prime for $i=$ $1, \cdots, m)$ is soluble. 
Then $G$ has a normal 2-complement.

Proof. By induction upon $|G|$.

First we note that, for each $p \in \pi(G) \backslash\{2\}, H$ contains all $\gamma$-invariant $p$-subgroups of $G$. By (2.3)(xiii) this is equivalent to showing that $H$ contains all $\gamma$-invariant Sylow $p$-subgroups of $G$, and, since $C_{G}(\gamma) \leqq$ $N_{G}(H)$ (by (ii) and (iii)), this follows from (i) and (2.3)(xiii). Similarly we have that $T$ contains all $\gamma$-invariant 2 -subgroups of $G$. Consequently, by (2.3)(xiii), $K=(K \cap T)(K \cap H)$ for all $\gamma$-invariant subgroups $K$ of $G$. Clearly $K$ will also satisfy conditions (ii) and (iii) with $K \cap T$ and $K \cap H$ in place of $T$ and $H$. Also observe that $\gamma$ invariant quotients of $G$ will satisfy conditions (i), (ii) and (iii). Hence we may assume that $O_{2^{\prime}}(G)=1$.

We assert that, if $G$ is soluble, then the conclusions of the lemma hold. For [Corollary $1 ; 6$ ] implies as $C_{G}(\gamma)$ is a 2-group, that $G=$ $N_{G}(J(T)) C_{G}(Z(T)) O_{2^{\prime}}(G)$. Then, by (iv) and [Theorem 8.1.1; 7], $G$ has a normal 2-complement. Therefore we may suppose $G$ is not soluble.

We now argue that $O_{2}(G)=1$. Suppose $O_{2}(G) \neq 1$, and let bars denote images of subgroups of $G$ in $G / O_{2}(G)$. Note that $\bar{T} \neq 1$, for otherwise $J(T)$ ch $T \leqq G$ implies that $G$ has a normal 2-complement. Therefore, since $Z(\bar{T}) \neq 1 \neq J(\bar{T})$, the inverse images in $G$ of $C_{\bar{G}}(Z(\bar{T}))$ and $N_{\bar{G}}(J(\bar{T}))$ must be proper $\gamma$-invariant subgroups of $G$ which contain $T$ and hence, by induction, will have normal 2-complements. Thus $N_{\bar{G}}(J(\bar{T}))$ and $C_{\bar{G}}(Z(\bar{T}))$ possess normal 2-complements. Applying induction to $\bar{G}$ yields $G=O_{2,2^{\prime}, 2}(G)$, which implies, by [4], that $G$ is soluble. Hence $O_{2}(G)=1$.

So $S(G)$ (the largest normal soluble subgroup of $G$ ) is trivial from which we infer

(a) $T$ is a maximal $\gamma$-invariant subgroup of $G$;

(b) $Z(T) \cap N_{T}(H)=1$; and

(c) $C_{Z(T)}(\beta)=1$ for all $\beta \in\langle\gamma\rangle^{\sharp}$.

Suppose $K$ is a proper $\gamma$-invariant subgroup of $G$ containing $T$. By induction $K$ has a normal 2-complement and so $O_{2^{\prime}}(K)=K \cap H$. Thus $(K \cap H)^{G}=(K \cap H)^{T H}=(K \cap H)^{H} \leqq H$ which forces $(K \cap H)^{G} \leqq$ $S(G)=1$. Hence $K=T(K \cap H)=T$ and this proves (a). Now $\left(Z(T) \cap N_{T}(H)\right)^{G} \leqq H N_{T}(H)$ and so $\left(Z(T) \cap N_{T}(H)\right)^{G} \leqq S(G)=1$, whence (b) holds. By (iii) (c) follows from (b).

By supposition $G$ is not soluble and so, from assumptions (ii) and $(\mathrm{v}), C_{T}(\gamma) \neq 1$. Let $x$ be an involution in $C_{T}(\gamma)$ and set $C=C_{G}(x)$. Clearly $C$ is a proper $\gamma$-invariant subgroup of $G$, and $C=(C \cap T)(C \cap H)$. By (c) and condition (ii) we have, using (2.8) upon $Z(T) O_{2^{\prime}}(C)$, $\left[Z(T), O_{2^{\prime}}(C)\right]=1$ whence (a) dictates that $O_{2^{\prime}}(C)=1$.

Suppose $C$ has a normal 2-complement. Then $C \cap H=O_{2^{\prime}}(C)=1$ 
and thus $x\left(\in N_{T}(H)\right)$ acts fixed-point-freely upon $H$. From (2.2)(i) $H$ is nilpotent and hence $G$ is soluble by (2.5). Thus we conclude that $C$ cannot have a normal 2-complement. Therefore $\mathscr{C} \neq \varnothing$, where $\mathscr{C}$ denotes the set of $\gamma$-invariant 2-subgroups $T_{1}$ of $T$ such that $N_{G}\left(T_{1}\right)$ does not have a normal 2-complement.

Among the elements of $\mathscr{C}$ choose $T_{1}$ such that the $\gamma$-invariant Sylow 2-subgroup of $N=N_{G}\left(T_{1}\right)$ has maximal order. We claim that $T \leqq N$. Suppose otherwise; so $R=T \cap N \neq T$. Since $Z(R)$ and $J(R)$ are characteristic subgroups of $R, N_{G}(J(R))$ and $C_{G}(Z(R))\left(\leqq N_{G}(Z(R))\right.$ will have normal 2-complements. Hence $N_{N}(J(R))$ and $C_{N}(Z(R))$ have normal complements and therefore so too has $N$ by induction. So $T \leqq N$, as claimed. But then, by (a), $T=N$ and so $T_{1} \notin \mathscr{C}$.

This concludes the proof of the lemma.

In Part IV we shall also require the following variation of Lemma 6.2 .

LEMMA 6.3. Suppose $G$ is a finite group admitting the coprime automorphism $\gamma$ of order st where $s$ and $t$ are distinct primes. Set $\gamma^{t}=\sigma$ and $\gamma^{8}=\tau$. Also suppose the following hold:

(i) $G=T H$ where $T$ is a $\gamma$-invariant Sylow 2-subgroup of $G$ and $H$ is a r-invariant Hall 2'-subgroup of $G$;

(ii) $H=H_{1} H_{2}$ where $H_{1}$ and $H_{2}$ are $\gamma$-invariant nilpotent Hall subgroups of $H$ with $H_{1_{\sigma}}=1=H_{2_{\tau}}$;

(iii) $C_{G}(\gamma)=C_{T}(\gamma)$;

(iv) $T_{o} \leqq N_{T}(H)$ and $T_{\tau} \leqq N_{T}\left(H_{1}\right)$; and

(v) $N_{G}(J(T))$ and $C_{G}(Z(T))$ both possess normal 2-complements. Then $G$ has a normal 2-complement.

Proof. By induction upon $|G|$. Note that (iii) and (iv) imply that $C_{G}(\gamma)=C_{T}(\gamma) \leqq N_{T}(H)$. As in Lemma 6.2 we may deduce that conditions (i), (ii), (iii) and (iv) hold for $\gamma$-invariant subgroups and $\gamma$-invariant quotients of $G$ and thence that $S(G)=1, T$ is a maximal $\gamma$-invariant subgroup of $G$ and $Z(T) \cap N_{T}(H)=1$. Hence, from (iv), $Z(T)_{\sigma}=1$. If $Z(T)_{\tau} \neq 1$, then, since $T_{\tau} \leqq N_{T}\left(H_{1}\right)$ and $H_{1_{\sigma}}=1$, $\left[Z(T)_{\tau}, H_{1}\right]=1$ by $(2.2)(\mathrm{i})$. But then $T, H_{1} \leqq C_{G}\left(Z(T)_{\tau}\right) \neq G$ forces $H_{1}=1$ which implies, by (2.5), that $G=S(G)=1$. Hence $Z(T)_{\langle a \tau\rangle}^{*}=1$. Since $S(G)=1$, (2.2)(ii) guarantees $C_{G}(\gamma) \neq 1$ and so we may show that $G$ has a normal 2-complement by imitating the latter part of the proof of Lemma 6.2.

LemMa 6.4. Suppose $G$ is a soluble group admitting the coprime fixed-point-free automorphism $\alpha$ of square-free order $r s t(r, s$ and $t$ primes). Set $\rho=\alpha^{s t}, \sigma=\alpha^{r t}$ and $\tau=\alpha^{r s}$, and let $H$ denote the 
$\alpha$-invariant Hall $\pi$-subgroup of G. If $H_{\rho}=H_{\rho_{\sigma}} H_{\rho_{\tau}}, H_{\sigma}=H_{\rho_{\sigma}} H_{\sigma \tau}$ and $H_{\tau}=H_{\rho_{\tau}} H_{o \tau}$, then $G$ has a normal $\pi$-complement.

Proof. Let $P$ denote the $\alpha$-invariant Sylow $p$-subgroup of $H$. Then, by (2.3)(i) and (ix), $P_{\rho}=P_{\rho_{\sigma}} P_{\rho_{\tau}}, P_{o}=P_{\rho_{o}} P_{o \tau}$ and $P_{\tau}=P_{\rho_{\tau}} P_{\sigma \tau}$, and so it will be sufficient to prove the lemma with $\pi=\{p\}$.

Deny the result and let $G$ be a counterexample of minimal order. Note that the lemma's hypotheses hold for $\alpha$-invariant subgroups of $G$ and $\alpha$-invariant quotients of $G$. So $O_{p^{\prime}}(G)=1$ by the minimal choice of $G$. Hence $C_{G}\left(O_{p}(G)\right) \leqq O_{p}(G)$ by [Theorem 6.3.2; 7], and we conclude that $G=O_{p}(G) Q$, where $Q$ is the $\alpha$-invariant Sylow $q$-subgroup of $G$ with $q \neq p$ and $Q$ possessing no nontrivial proper $\alpha$ invariant subgroups. Furthermore, the minimality of $G$ together with (2.3)(iii) implies that $O_{p}(G)$ is a minimal normal $\alpha$-invariant subgroup of $G$. Therefore $O_{p}(G)$ is an elementary abelian $p$-group and $C_{o_{p}(G)}(Q)=1$.

From (2.10)(ii) we deduce that $\left[O_{p}(G)_{\rho}, Q_{\rho}\right]=\left[O_{p}(G)_{\sigma}, Q_{\sigma}\right]=$ $\left[O_{p}(G)_{\tau}, Q_{\tau}\right]=1$. Moreover $O_{p}(G)^{*} \neq 1 \neq Q^{*}$ by (2.8). So we may suppose $Q_{\rho} \neq 1$. Since $Q_{\rho}$ is $\alpha$-invariant, $Q=Q_{\rho}$ and so $O_{p}(G)_{\rho} \leqq$ $C_{o_{p}(G)}(Q)=1$. Consequently $O_{p}(G)^{*}=O_{p}(G)_{\sigma}=O_{p}(G)_{\tau} \neq 1$. Hence $Q_{\sigma}=$ $Q_{\tau}=1$. So $Q\langle\sigma \tau\rangle$ is a Frobenius group which is faithfully and irreducibly represented upon $O_{p}(G)$. By a well-known result, this representation when restricted to $\langle\sigma \tau\rangle$ contains the regular representation of $\langle\sigma \tau\rangle$ and, under these circumstances, $O_{p}(G)_{\sigma}=O_{p}(G)_{\text {- }}$ cannot hold.

Hence there does not exist a counterexample, and the lemma is verified.

\section{REFERENCES}

1. T. R. Berger, Nilpotent fixed-point-free automorphism groups of solvable groups, Math. Z., 131 (1973), 305-312.

2. — Hall-Higman type theorems VII, Proc. London Math. Soc., (3) 31 (1975), 21-54.

3. C. W. Curtis and I. Reiner, Representation Theory of Finite Groups and Associative Algebras, Wiley-Interscience, New York, 1962.

4. W. Feit and J. G. Thompson, Solvability of groups of odd order, Pacific J. Math., 13 (1963), 775-1029.

5. G. Glauberman, Fixed points in groups with operator groups, Math. Z., 84 (1964), 120-125.

6. - Failure of factorization in p-solvable groups, Quart. J. Math., Oxford (2), 24 (1973), 71-77.

7. D. Gorenstein, Finite Groups, Harper and Row, New York, 1968.

8. M. Hall and J. K. Senior, The Groups of $\operatorname{Order} 2^{n}(n \leqq 6)$, Macmillan, New York, 1964,

9. B. Huppert, Endliche Gruppen I, Springer-Verlag, Berlin, 1967.

10. R. P. Martineau, Elementary abelian fixed-point-free automorphism groups, Quart. J. Math., Oxford (2), 23 (1972), 205-212.

11. R.P. Martineau, Solubility of groups admitting certain fixed-point-free automorphism 
groups, Math. Z., 130 (1973), 143-147.

12. R. P. Martineau, unpublished.

13. M. R. Pettet, Fixed-point-free automorphism groups of square-free exponent, Proc. London Math. Soc., (3) 33 (1976), 361-384.

14. E. W. Ralston, Solvability of finite groups admitting fixed-point-free automorphisms of order rs, J. Algebra, 23 (1972), 164-180.

15. P. J. Rowley, Finite groups which admit a fixed-point-free automorphism of order rst, Warwick University preprint, (1975).

16. - Solubility of finite groups admitting a fixed-point-free abelian automorphism group of square-free exponent rs, Proc. London Math. Soc., (3) 37 (1978), 385-421.

17. - Finite groups admitting a fixed-point-free automorphism of square-free order, (in preparation).

18. E. E. Shult, On groups admitting fixed-point-free abelian operator groups, Illinois J. Math., 9 (1965), 701-720.

19. J. G. Thompson, Finite groups with fixed-point-free automorphisms of prime order, Proc. Nat. Acad. Sci., U.S.A., 45 (1959), 578-581.

Received January 5, 1979 and in revised form June 23, 1980.

UNIVERSity OF Birmingham

P.O. Box 363

Birmingham B15 2TT

ENGLAND 



\section{PACIFIC JOURNAL OF MATHEMATICS}

\section{EDITORS}

DONALD BABBITT (Managing Editor)

University of Galifornia

Los Angeles, California 90024

HUgo RossI

University of Utah

Salt Lake City, UT 84112

C. C. MOORE AND ANDREW OGG

University of California

Berkeley, CA 94720

\section{J. DUGUNDJI}

Department of Mathematics University of Southern California Los Angeles, California 90007

R. Finn and J. Milgram

Stanford University

Stanford, California 94305

\section{ASSOCIATE EDITORS}
R. ARENS
E. F. BECKENBACH
B. H. NeUManN
F. WOLF
K. YosHIDA

\section{SUPPORTING INSTITUTIONS}

UNIVERSITY OF ARIZONA

UNIVERSITY OF BRITISH COLUMBIA

CALIFORNIA INSTITUTE OF TECHNOLOGY

UNIVERSITY OF CALIFORNIA

MONTANA STATE UNIVERSITY

UNIVERSITY OF NEVADA, RENO

NEW MEXICO STATE UNIVERSITY

OREGON STATE UNIVERSITY
UNIVERSITY OF OREGON UNIVERSITY OF SOUTHERN CALIFONIA STANFORD UNIVERSITY UNIVERSITY OF HAWAII UNIVERSITY OF TOKYO UNIVERSITY OF UTAH WASHINGTON STATE UNIVERSITY UNIVERSITY OF WASHINGTON 


\section{Pacific Journal of Mathematics}

\section{Vol. 93, No. $1 \quad$ March, 1981}

Richard Arens, Reducing the order of a Lagrangian $\ldots \ldots \ldots \ldots \ldots \ldots \ldots$

Richard Arens, Manifestly dynamic forms in the Cartan-Hamilton treatment

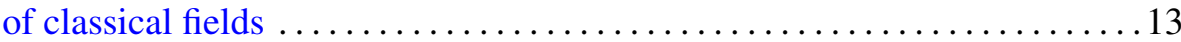

Jimmy T. Arnold, Power series rings over discrete valuation rings $\ldots \ldots \ldots 31$

Charles A. Asmuth and Joe Repka, Supercuspidal components of the

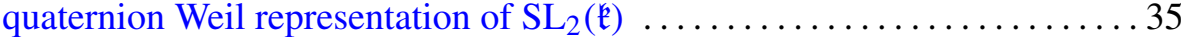

Luis A. Caffarelli and Avner Friedman, Sequential testing of several simple hypotheses for a diffusion process and the corresponding free boundary problem ................................. 49

William B. Jacob, Fans, real valuations, and hereditarily-Pythagorean fields .............................................. 95

W. J. Kim, Asymptotic properties of nonoscillatory solutions of higher order

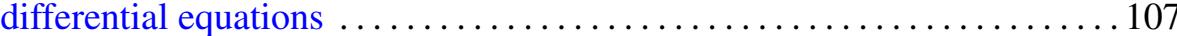

Wayne Steven Lewis, Embeddings of the pseudo-arc in $E^{2} \ldots \ldots \ldots \ldots \ldots 115$

Daniel Alan Marcus, Closed factors of normal Z-semimodules . ......... 121

Mitsuru Nakai and Leo Sario, Harmonic functionals on open Riemann surfaces ............................................. 147

John Currie Quigg, Jr., On the irreducibility of an induced

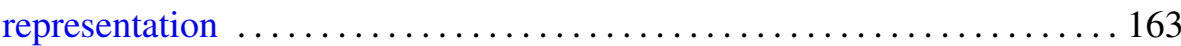

John Henry Reinoehl, Lie algebras and Hopf algebras 181

Joe Repka, Base change for tempered irreducible representations of $\mathrm{GL}(n, \mathbf{R})$

Peter John Rowley, Solubility of finite groups admitting a fixed-point-free automorphism of order $r s t$. I . . . . . . . . . . . . . . . . . . 201

Alan C. Woods, The asymmetric product of three homogeneous linear forms 